\title{
The Tight Coupling and Non-Linear Relationship between the Macroscopic Electrical and Optical Concomitants of Electrochemical CNS Waves Reflect the Non-Linear Dynamics of Neural Glial Propagation
}

\author{
Vera Maura Fernandes de Lima1,2, José Roberto Castilho Piqueira ${ }^{3}$, Wolfgang Hanke \\ ${ }^{1}$ CCO Medical School, Universidade Federal de São João Del Rei, Sao Joao Del Rei, Brazil \\ ${ }^{2}$ LIM-26 Faculty of Medicine, USP, São Paulo, Brazil \\ ${ }^{3}$ Laboratory of Automation and Control, Escola Politecnica, USP, São Paulo, Brazil \\ ${ }^{4}$ Membrane Physiology Division, Physiology Institute 230, Hohenheim University, Stuttgart, Germany \\ Email: wolfgang.hanke@uni-hohenheim.de
}

Received 8 September 2014; revised 7 October 2014; accepted 6 November 2014

Copyright (C) 2015 by authors and Scientific Research Publishing Inc.

This work is licensed under the Creative Commons Attribution International License (CC BY).

http://creativecommons.org/licenses/by/4.0/

C. (7) Open Access

\begin{abstract}
In isolated chick retina, the visualization of electrochemical self-organized patterns is possible due to the presence of macroscopic intrinsic optical signals (IOSs). Isolated circular waves, standing patterns, and self-sustained sequences of spirals are all easily obtained using an IOS approach. In this paper we present the tight coupling and non-linear relationship between optical and electrical wave concomitants, and potassium-induced whole tissue excitability changes. Elementary statistical methods and time series analyses were applied to two sets of data: 1) solitary circular retinal spreading depression waves, and 2) tissue response to exogenous potassium fast pulses. The results were interpreted from the point of view of non-linear thermodynamical concepts and volume phase transitions in polyanionic gels according to the Tasaki action potential model. From these and previous results, it is clear that the glial network and extracellular matrix contribute to the propagation and emergence of these patterns.
\end{abstract}

\section{Keywords}

Glial Network, Extracellular Matrix, KCl, Spreading Depression, Excitable Media 


\section{Introduction}

Solitary, circular retinal spreading depression (RSD) waves are concurrently: 1) an example of a two-dimensional excitation wave propagating in excitable media [1]; 2) a self-organized two-dimensional electrochemical pattern [2]; and 3) a model for functional syndromes of the central nervous system (CNS) [3]-[5]. Results from the RSD experimental model can therefore have an interdisciplinary appeal for a range of researchers, from theorists to physicians to medical image processing physicists.

In this paper we show that field potentials and intrinsic optical signals (IOS) recorded at inner retina (inner plexiform layer and vitreal border, respectively) are tightly coupled wave concomitants. Our results, however, did not reveal any linear causality between them. The IOS and field potential time series were recorded simultaneously and optical changes were recorded at microscopic scale as close as possible to the electrode tip. IOS and field potential time derivative peaks coincided in time in 44 waves of RSD recorded from 34 retinas.

When a propagating wave front invades the region around the electrode tip, the field potential of RSD waves at the inner plexiform layer is usually dominated by a single potential drop. A single peak therefore dominates the time derivative of the field potential time series recorded during passage of solitary circular wave fronts. In the CNS of retinas, the external leaflets of glial and synaptic membranes-which are rich in negatively charged polymers such as gangliosides or glycolipids, proteoglycans, and glycoproteins-interact through the negatively charged gel (glycosaminoglycans [GAGs]) of extracellular space at the inner plexiform layer. The non-linear neuronal/glial interaction in this system has been demonstrated at both membrane-channel and intracellular levels [6]-[9] during light responses and RSD waves. The results presented here are interpreted from this point of view for two sets of data: from 44 circular solitary spreading depression waves from 34 retinas, and seven fast high-concentration exogenous $\mathrm{KCl}$ pulses recorded in five retinas.

Aside from circular solitary waves elicited by a light touch, $\mathrm{KCl}$ solutions are a classic trigger for RSD waves and other electrochemical self-organized patterns, for example, as used for experimental hippocampal seizures. With only a few pulse experiments, we recorded the full range of optical and field potential changes associated with exogenous $\mathrm{KCl}$ that have been described in the RSD literature [4] [10]-[13].

Finally, we show that when a solitary front invades a patch of attached retina viewed from the side, the optical changes occur first at the inner plexiform layer and an abrupt loss of transparency then marks the tissue's change from a quiescent to excited state. These results confirmed the early observations of Martins-Ferreira and Oliveira e Castro about the source of the IOS during RSDs as well as more recent results [14] [15] obtained from confocal microscopes that measured light scatter in undyed live scotopic retinas. In our case an ordinary optical microscope was used, with the retina illuminated with laser light.

The main objective in our discussion of the results is to demonstrate the importance of the glial network geometry in the shape of electrochemical waves and the probabilities of other spatial patterns. Additionally, in the particular case of retinas, we want to show that the glial network geometry plays an important role in the origin of the IOS associated with these electrochemical patterns. We propose that in the retinal model, the informational role of the polyanionic gel of the basement membranes and extracellular matrix [16]-[19] can be observed directly in the RSD wave propagation.

\section{Materials and Methods}

\subsection{Preparation of the Eye-Cup}

Chicken at the age of 5 to 21 days were used for the experiments. Chicken were bought directly after hatch and kept in a proper stable at the University of Hohenheim until the day of the experiment, and killed by decapitation. After decapitation, the eyes were removed from the eye socket. Eyes were sectioned close to the equator with a razor blade and the vitreous body was removed with tweezers. The posterior eye-cups were immersed in Ringer solution. The eye-cups were then glued in individual Petri dishes and placed in the setup, where they were perfused with Ringer solution. Before we started collecting measurements, the retinas were allowed to recover for $30 \mathrm{~min}$. The keeping of chicken and all associated experiments were performed within the related laws for Germany and the EU.

\subsection{Ringer Solution}

The solution used to perfuse the isolated retinas had the following composition: $100 \mathrm{mM} \mathrm{NaCl}, 6 \mathrm{mM} \mathrm{KCl}, 1$ 
$\mathrm{mM} \mathrm{MgSO}_{4}, 1 \mathrm{mM} \mathrm{H} \mathrm{H}_{2} \mathrm{PO}_{4}, 1 \mathrm{mM} \mathrm{CaCl} \cdot 2 \mathrm{H}_{2} \mathrm{O}, 30 \mathrm{mM} \mathrm{NaHCO}, 10 \mathrm{mM}$ TRIS, and $30 \mathrm{mM}$ glucose (pH 7.4). The temperature of the experiments was $30^{\circ} \mathrm{C}$.

\subsection{Retina Setup 1}

The setup was enclosed in a Faraday cage on a vibration isolation table (Halcyonics MOD-1). A binocular microscope (Leica MS5) equipped with two beam splitters was used for observation of the retinas. To one of the beam-splitters was connected an RGB-camera (Sony Power HAD), and to the other, a photomultiplier (RCA 6199 with a Hamamatsu adapter and a Physic Instruments high voltage power supply). Electrical recordings were performed with extracellular glass micro-electrodes (tip diameter around $20 \mu \mathrm{m}$, pulled on a Zeitz puller) filled with Ringer solution, see above. These were inserted in the retina using a micromanipulator (Eppendorf PatchMaster) under optical control. The positioning of the measuring electrode was aimed at the inner plexiform layer. The sharpness of field potential laminar profiles within retinas makes it easy to recognize the electrode tip position (Fernandes de Lima and Hanke, 1997). The electrical potential of the tissue relative to the bathing solution was measured with a high impedance amplifier (WPI double channel electrometer FD223) connected to an $\mathrm{Ag} / \mathrm{AgCl}$ electrode in the glass micropipette versus another $\mathrm{Ag} / \mathrm{AgCl}$ coil wire electrode immersed in the bath (reference electrode).

\subsection{Retina Setup 2}

In Figure 1 we show the experimental setup used in the records shown in Figure 10 and Figure 11. In the figure we show (clockwise): a) The combined output from the two cameras. They were connected to two optical
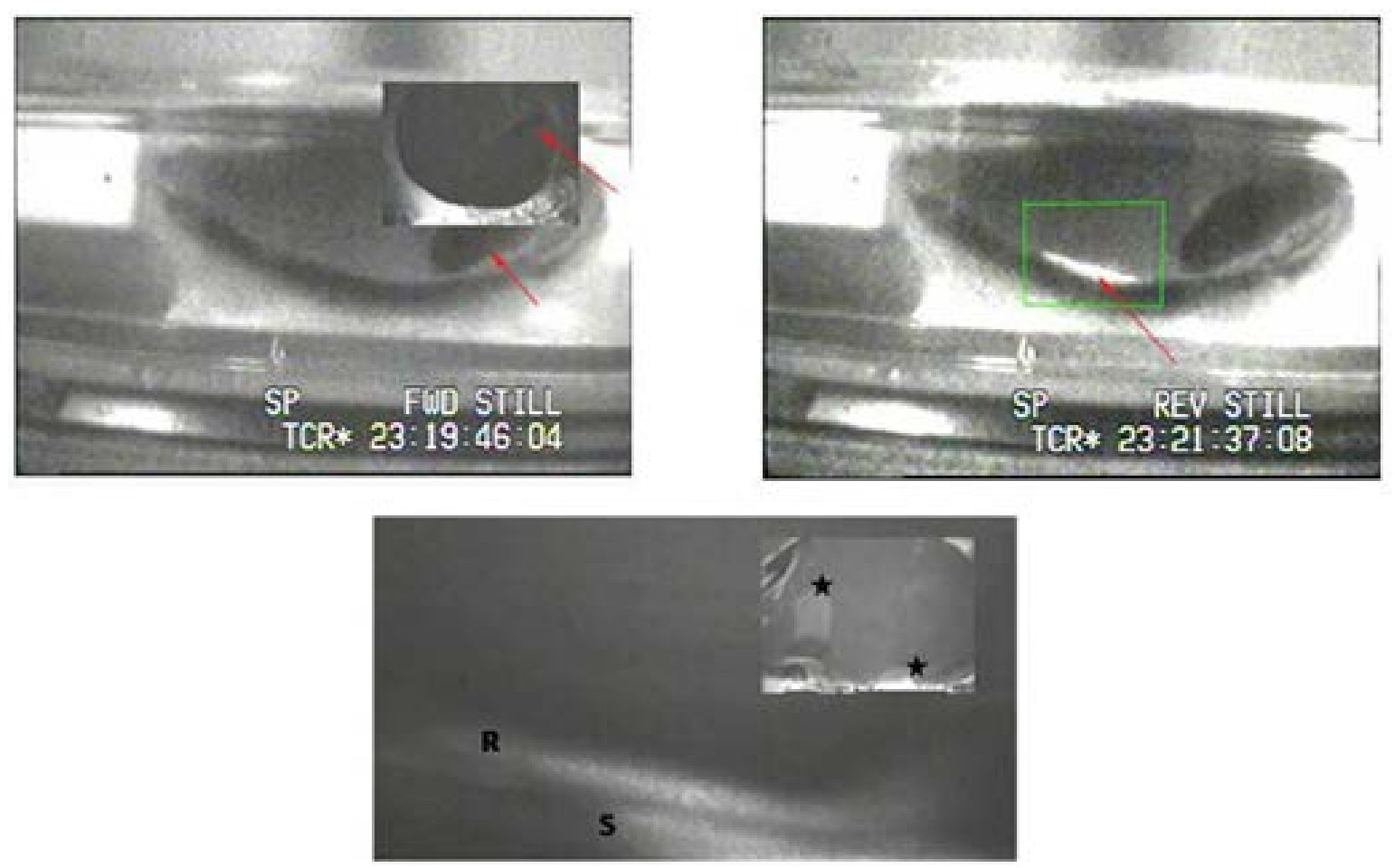

Figure 1. Clockwise: The three photos show the experimental recording setup used to record the RSD IOS along the width of in situ retinas. The first photo shows the combined images (marked by arrows) of the two cameras. The camera positioned above the retina provides a panoramic view of the retinal surface. The second camera is positioned at $90^{\circ}$ from the first and provides a lateral view of the in situ retina. The second photo shows the area illuminated with laser beam. The beam was positioned at $45^{\circ}$ from the retinal surface and aimed at the border. The length of the pecten is $6.5 \mathrm{~mm}$. The last photo shows the outputs of the two cameras during a record whereby a wave was elicited and a second wave arose "spontaneously" within the area illuminated with the laser (marked by asterisks). The gain of the second microscope amplification was smaller than the one shown in Figure 10 and Figure 11. In this image, a part of the sclera (denoted S) contributes to the light scatter. The "spontaneous" wave could be seen propagating within the retina (denoted R). 
microscopes (Leica camera 1 and Olympus camera 2) and their outputs were combined in a single frame before recording with a video editor; b) The second camera was positioned at 90 degrees from the first and provided a view of the retina width. A laser beam $(640 \mathrm{~nm}, 5 \mathrm{~mW})$ was positioned at the central retina. The second microscope was focused on the illuminated area (region inside the square in the figure); and c) The first camera provided a panoramic view of the retina surface viewed from above. The last photo shows an elicited RSD wave propagating in the first camera and another that "spontaneously" aroused within the laser-illuminated area (marked by asterisks). In the second camera, the retina (denoted R) and parts of the sclera and Petri (denoted S) dish scattered the laser beam.

\subsection{Measuring Software}

Data from retina setup 1 were digitized and stored on a computer using home-written routines in LabView ${ }^{\circledR}$ together with proper A/D-converter hardware. The photomultiplier and electrode outputs were stored simultaneously as a time series, while the camera output (via a NI video-grabber card) was stored as a video file (video sampling rate 25 full frames per second, PAL solution).

The electrode output and the mean brightness of a $10 \times 10$ pixel matrix $(50 \mu \mathrm{m}$ side length $)$ that was positioned over the electrode tip within the video frames and sampled simultaneously with the photomultiplier output and electrode/amplifier system output at $10 \mathrm{~Hz}$ were stored on a computer and visualized on a display in real time. The photomultiplier output measured optical profiles at macroscopic scale over a $1 \mathrm{~mm}$ diameter circular area.

Typical time series are shown in Figure 2. The figure shows raw data and the time derivative of the field potential time series obtained using ORIGIN ${ }^{\circledR}$ software off-line.

The figure shows two optical profiles obtained at different spatial scales and the field potential measured in the inner plexiform layer of in vitro retinas. All graphs are at the same time scale to show a complete optical profile. Note that the field potential is correlated with the first optical component of the profile, whereas the second is related to the metabolic tissue response.

\subsection{Processing of Video Frames}

The data in Figure 10 and Figure 11 were processed in the following way: the output from the two cameras was combined before recording, and samples of the video-recorder data were digitized as gray scale. The first frame of a video was used as background and the pixel brightness value subtracted from each subsequent frame. In the figure, the brightness is displayed in false colors. ImageJ software was used to process the video frames.

\subsection{Materials}

All chemicals were obtained at least at p.a. grade from Sigma, Aldrich or Merck. Water was from a laboratory distillery.

\section{Results}

\subsection{General Statements about RSD Waves, Their Optical Profiles, and Field Potentials}

All waves examined in this study were solitary circular waves that had been mechanically elicited by a light touch. Recent panoramic views of such waves and their optical profiles are available in the literature [19] [20]. RSD waves are characterized by their circular shape and smooth continuous invasion of quiescent tissue, which separates them from other forms of propagating excitation such as the excitotoxic response [21]. At this point, it should be noted that the optic nerve exit, or papilla, does not interfere with either the shape or the intensity of IOS. However, the structure of the retinal tissue is changed drastically: there are no plexiform or nuclear layers, only the radial glia and exiting axons [22] [23]. From excitable media theory it is known that wave curvature and propagation velocity are tightly coupled variables [24]. Thus, the drastic change in retinal tissue structure does not interfere with wave propagation. This observation has been described since the first report of Martins-Ferreira [25].

Figure 2 shows the optical profiles at two different spatial scales. With the exception of the sudden onset at the microscopic scale, both profiles are similar. For 44 RSD profiles (measured at macroscopic scale) using data 

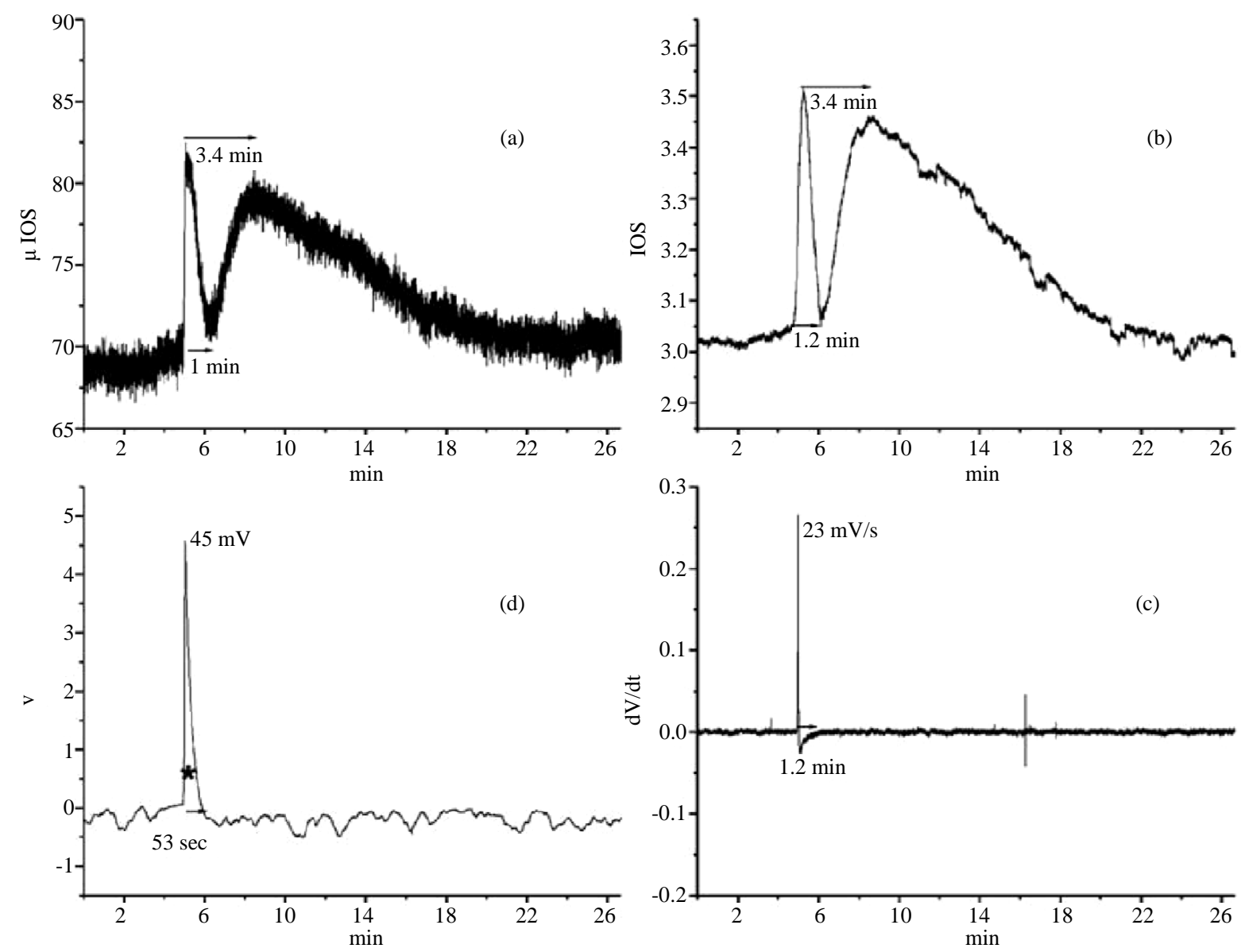

Figure 2. (a) and (b) are typical optical profiles of circular RSD waves, recorded at micro and macro space scales at $30^{\circ} \mathrm{C}$. (c) is the time derivative of the field potential time series. (d) shows the concomitant potential drop within the inner-plexiform layer. In (a) the ordinate in arbitrary units shows the mean brightness of a $10 \times 10$ pixel matrix (about $50 \mu \mathrm{m}$ side-length). The graph in (b) shows the digitized output of a photomultiplier that samples a circular area $1 \mathrm{~mm}$ in diameter. The output is proportional to the light scattered by the tissue and it is again in arbitrary units. The arrows in (a) and (b) show the time interval between the first and second optical peaks of the same wave profile, as well as the duration of the first peak in relation to the electrochemical variables of the RSD wave. In (c), the $\mathrm{dV} / \mathrm{dt}$ time series of the field potential is given, where the shape is typical for a solitary circular RSD wave. Peak amplitude and the duration of the transient are shown in the graph. In (d), the field potential was measured with a glass micro-electrode $(10 \mu \mathrm{m}$ tip diameter) filled with $\mathrm{KCl}$ solution inserted in the inner-plexiform layer against a silver/silver chloride coil immersed in the Petri dish. The amplifier output was filtered at 30 $\mathrm{Hz}$ ( $-6 \mathrm{~dB}$ /octave). Peak amplitude and the time of recovery to baseline are shown in the figure. The asterisk indicates the position of the $\mathrm{dV} / \mathrm{dt}$ peak. The abscissas are in minutes. Data acquisition and processing were performed in LabView ${ }^{\circledR}$ and ORIGIN $^{\circledR}$, respectively.

from 34 retinas, the duration of the optical response was measured, as well as the area under the curve for the total profile and the second or metabolic component (measured from the minimum between the peaks).

Profile duration ranged from 6.6 to 23.87 minutes (mean $=15.3$, sd $=3.9$ ) of which the second component represented $82.3 \%$ to $94.2 \%$ of the total area (mean $=89.6$, $\mathrm{sd}=2.68$ ). Two profiles, which accounted for $4 \%$ of the waves, had a first optical component of smaller amplitude than the second component. In these waves the second component represented 94\% of the total area. However, other parameters, such as the interval between peaks and the duration of the first peak, were as expected for solitary RSD waves measured at $30^{\circ} \mathrm{C}$. Figure 2 shows the time interval between the two optical peaks of each profile, the width of the first optical component, and the field potential of the wave. Our experimental values are typical of solitary circular waves obtained from quiescent retinas at $30^{\circ} \mathrm{C}$. The asterisk in the field potential graph indicates when the peak of the $\mathrm{dV} / \mathrm{dt}$ time series occurs. Since this peak coincides with the peak of the dK/dt profile [13], the rising phase of the field potential is associated with the dissipation of electrochemical gradients. Meanwhile, the recovery phase is associated 
with the acceleration of the sodium potassium pump and energy consumption in the tissue. The inter-peak interval is the period when the tissue is at absolute refractoriness. The relative refractoriness, on the other hand, has the duration of the total optical profile [26]-[28].

The rise of the second optical peak is closely associated with a pH change (acid shift in the extracellular space) due to glia secretion of lactic acid [29]. This is because in the isolated avascular retina, metabolism depends up to $100 \%$ on glial glycolysis [14]. The second optical peak also shows an analogue of the metabolic response of the tissue to the challenge of the synchronous electrochemical gradient dissipation at the wave onset.

\subsection{Descriptive Statistics of Field Potentials and Their Relationships with the Micro-Scale IOS}

Figure 3 shows the electrophysiological and optical concomitant signals for one of the 44 RSD waves from 34 retinas measured.

The simultaneously recorded time series are displayed at the same temporal scale of 10 minutes. The complete optical profile shown in Figure 2 has a total duration close to 20 minutes. Figure 3 shows the RSD wave concomitant signals using an expanded scale of 10 minutes, in order to show details of the onset phase. In Figure 3(d), the field potential and its time derivative are shown. The amplitude distribution of the 44 field potential waves concomitant with RSD waves is shown in Figure 3(b), while 3C shows the scatter of the points that correlate the field potential amplitude with the amplitude of its time derivative. A strong (0.88) linear correlation was found between the two signals. The same degree of correlation and scatter has also been found between the
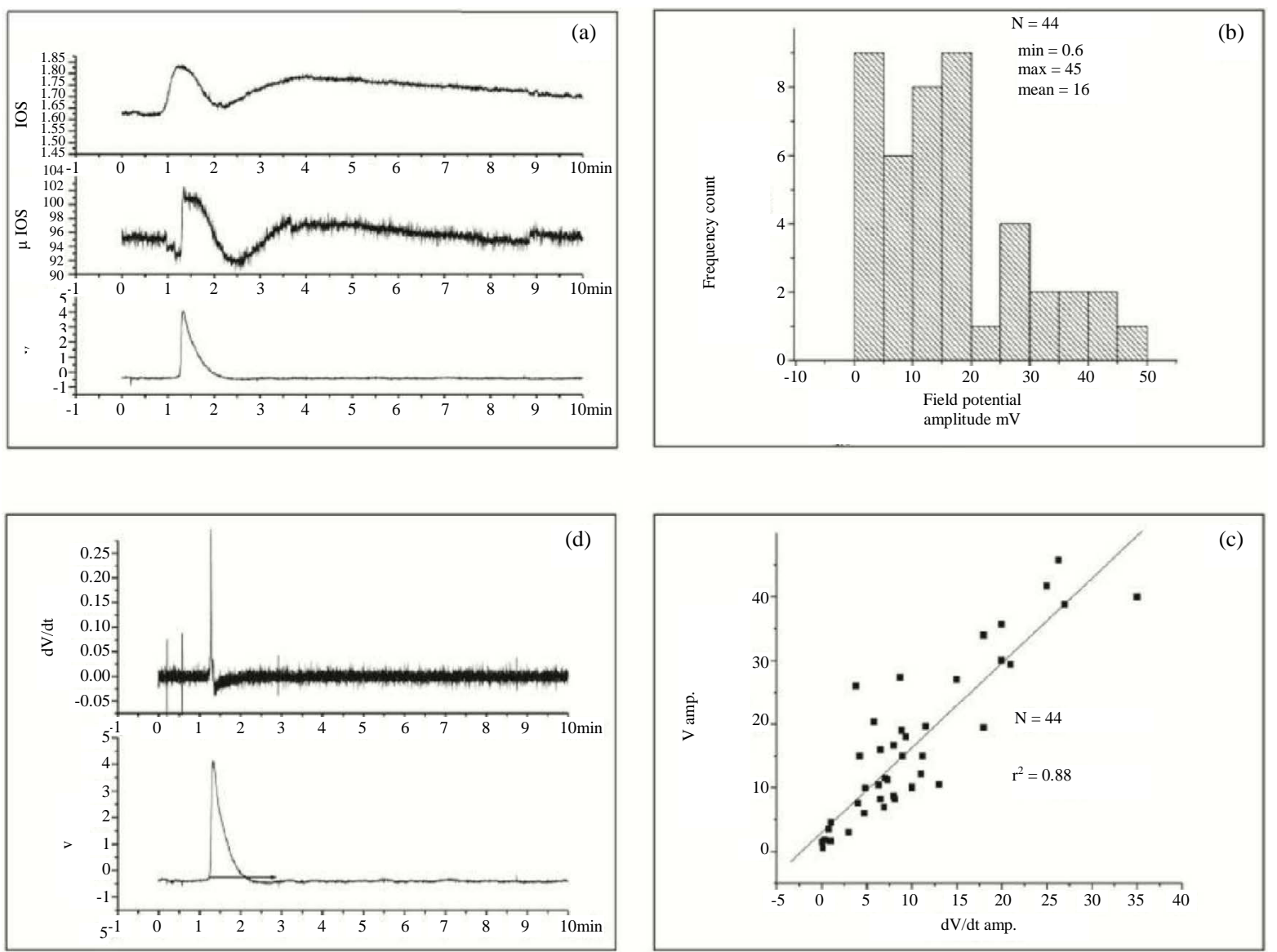

Figure 3. In (a), the three simultaneously time series are shown on an expanded temporal scale (10 minutes). In (b) the bar diagram shows the frequency count of the field potential amplitude for 44 RSDs. (c) shows the scatter plot of the field potential amplitude versus the $\mathrm{dV} / \mathrm{dt}$ peak amplitude. Linear regression and correlation coefficient are depicted in the graph. (d) has the field potential and dV/dt time series used to create graph C. Note the shape of the field potential with a characteristic undershoot in the after-potential. 
amplitude of the derivative and the velocity of wave propagation [30]. The range of field potentials was 0.6 to $45 \mathrm{mV}$ with a mean of $16 \mathrm{mV}$. The range of the time derivative peak was 0.3 to $37 \mathrm{mV} / \mathrm{s}$ with a mean of 12.8 $\mathrm{mV} / \mathrm{s}$. The smallest $(0.6 \mathrm{mV})$ amplitude also had a shape different from the ones shown in the previous figures: double peak kinetics with a long duration, taking 1.5 minutes to return to baseline. We note that this shape has been published before together with the associated potassium activity [4]. In one wave, we could identify a 200 $\mu \mathrm{V}$ sharp potential drop that coincided with the peak of the time derivative of the micro-scale IOS. This wave could be classified as a "zero-field potential wave" depending on the amplifier setup. These two waves had micro-scale IOSs that were no different from the ones shown in Figures 2-4.

The relationship between the RSD wave's electrophysiological and optical concomitant signals is then nonlinear, as can be seen in the figures. The close association of their maximum rate of rising suggests a tight coupling between the change in ionic environment change within the extracellular hydrogel, and the fast change in optical properties of the tissue.

\subsection{Field Potential and Micro Optical Profiles of Exogenous Potassium Pulses}

Potassium is the universal depolarizing agent and inducer of exocytosis for all excitable tissues, from CNS nerve terminals to endocrine glands. It has also been long known that exogenous $\mathrm{KCl}$ produces spreading depression waves in grey matter and reversibly changes the optical properties of retinas [10] [31]. If a salt solution is added
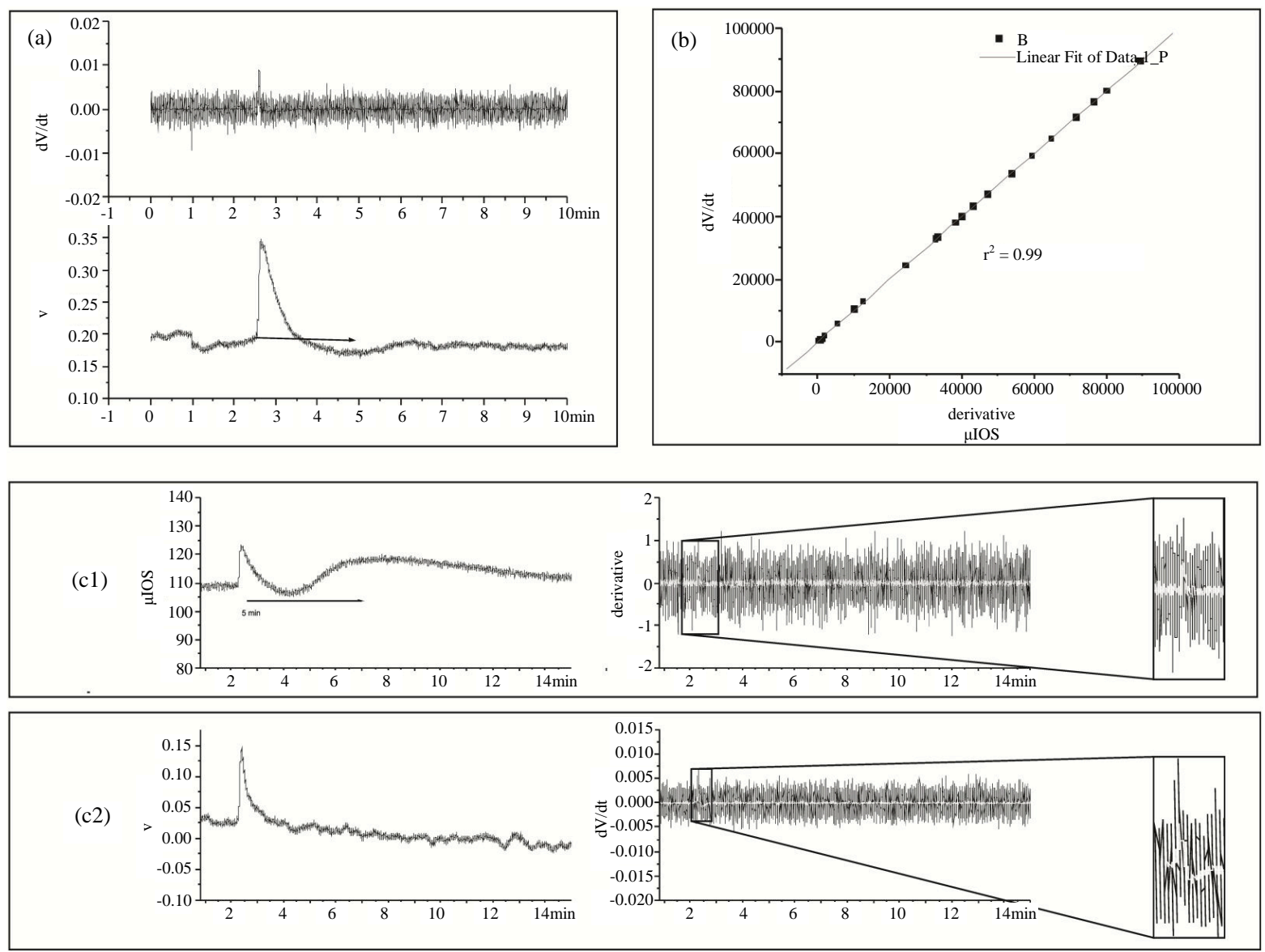

Figure 4. In (a) the low amplitude field potential and dV/dt time series are given. Note the shape conservation for the field potential and the decrease in the signal to noise ratio for the $\mathrm{dV} / \mathrm{dt}$ peak amplitude. (b) shows a scatter plot of the time of occurrence for the temporal derivatives for the field potential and micro-scale IOS peaks. The linear regression line and correlation coefficient are depicted on the graph $(\mathrm{n}=44)$. (c1) and (c2) present the time series of micro-scale IOS and field potential for a RSD wave with low amplitude field potential $(3.5 \mathrm{mV})$ and long inter-peak interval in the optical profile (5 min). This value is twice the expected length at $30^{\circ} \mathrm{C}$. The time derivative is shown to the side of each time series. The output of a low-pass adjacent-averaging filter is superimposed on the original series and the expanded peaks in the insets. 
to the perfusion system without disturbance of the laminar flow, potassium activity rises slowly within the inner plexiform layer (IPL) and tissue transparency increases until a wave is triggered [13]. Before the wave, the rise in potassium activity does not change the field potential recorded within the IPL. By contrast, a sudden rise in potassium activity at the IPL occurs either "spontaneously" [4] or as a result of ouabain application [21] [26], and decreases the transparency of the tissue and produces a potential drop. When a RSD wave is triggered, the time derivative of the field potential and the abrupt rise in potassium activity wave coincide in time [13]. In the previous sections, we showed that the optical signal associated with RSD waves also coincide in time with the field potential time derivative. In this section we will show that changes in optical properties and field potential elicited by fast, high concentration pulses of $\mathrm{KCl}$ aspersed over the inner-limiting membrane.

Seven pulses from five retinas were used in the experiments. Figure 5 and Figure 8 show two alternative ways of summarizing the complete experimental data: 1 ) the three simultaneously recorded time series already shown in the previous section that shows temporal evolution of the optical changes and field potential; and 2) the time/space evolution of the optical changes in a different retina depicted using the kymograph technique. The latter shows how widespread these optical changes were in central retina.

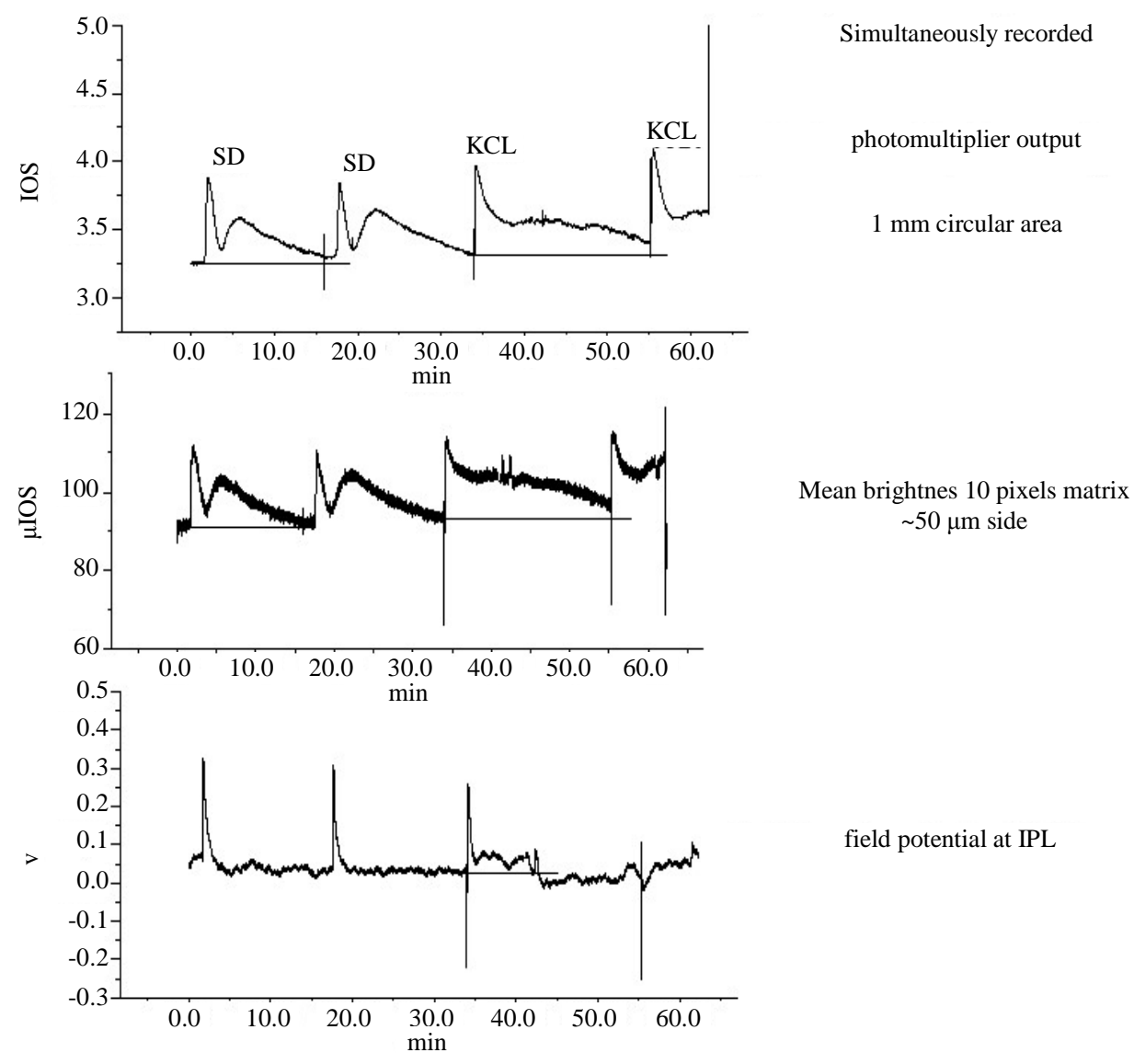

Figure 5. The three simultaneously recorded time series in a $\mathrm{KCl}$ short pulse experiment. The total time of the record is 62 minutes. Top: photomultiplier output time series. The macroscopic $(1 \mathrm{~mm})$ optical profiles of two circular spreading depression waves were recorded before the first $\mathrm{KCl}$ pulse response after pulses of $200 \mu \mathrm{l} / 250 \mathrm{mM} \mathrm{KCl}$ solution. Note a sharp hyper-transparency phase in both pulse responses, which is much smaller in the second pulse. The optical profile of the first response has a prolonged plateau that was still not completely back to baseline transparency level 21 minutes and 20 seconds later when the second pulse was applied. The optical profile of the second pulse response was recorded for 7 minutes. Center: the microscopic optical signal recorded very close to the electrode tip at the retinal surface $(10 \times 10$ pixel matrix mean brightness). From the movie and this time series, the optical artifact produced by reflections and shadows of the pipette and flow turbulence caused by the pressure pulse could be estimated at 1.2 seconds for both pulses. Note that at this small patch of retina, both pulses produced hyper-transparency that preceded the excitation associated with increase in light scatter. Bottom: field potential time series. Note the slow negative shift of the baseline after the first pulse (duration 8 minutes) and the absence of shift in field potential after the second pulse. 
It should be noted that in Figure 8 the recording electrode tip is covered in the digital frame by the superimposed time series and that it rests about $1 \mathrm{~mm}$ above and to the right of the center of the frame. With pressure ejection of $\mathrm{KCl}$ solutions, very high $(1$ and $2 \mathrm{M})$ concentrations have been used in spreading depression experiments: for example see, [11] [32] [33]. In all these reports brief $\mathrm{KCl}$ pulses did not cause any visible damage to cortices, hippocampal slices, or in vitro retinas. In the submerged in vitro retina, $1 \mathrm{M} \mathrm{KCl}$ application very close to the vitreal border (5 seconds) was used for the record shown by the authors [11]. We used 100 and $200 \mu$ volumes of a $250 \mathrm{mM} \mathrm{KCl}$ solution (see methods section) ejected with Eppendorf pipettes that were held close to the vitreal border of the central retina.

Figure 5 shows the simultaneously recorded time series in one experiment in which two RSD wave recordings preceded two exogenous potassium pulses $(200 \mu \mathrm{l} / 250 \mathrm{mM})$. In Figure 5 we can see that the optical profile of a $\mathrm{KCl}$ excitation wave can have a different profile from the RSD waves elicited by mechanical stimulation that are far away ( $\sim 3$ to $5 \mathrm{~mm}$ ) from the recording point. There is no clear distinction between the electrochemical and metabolic peaks but plateaus of increased light scatter that fall slowly to the baseline level of tissue transparency are present. The field potential also has a sharp initial component and a small shift of the baseline to relative negative potential in the first pulse, and much faster kinetics following the second pulse that was delivered 20 minutes after the first. In Figure 6 and Figure 7, the optical changes close to the electrode tip and the field potentials elicited by these two $\mathrm{KCl}$ pulses are shown in a highly expanded time scale in order to follow the optical changes and field potentials in the critical transition from stimulation to wave propagation.

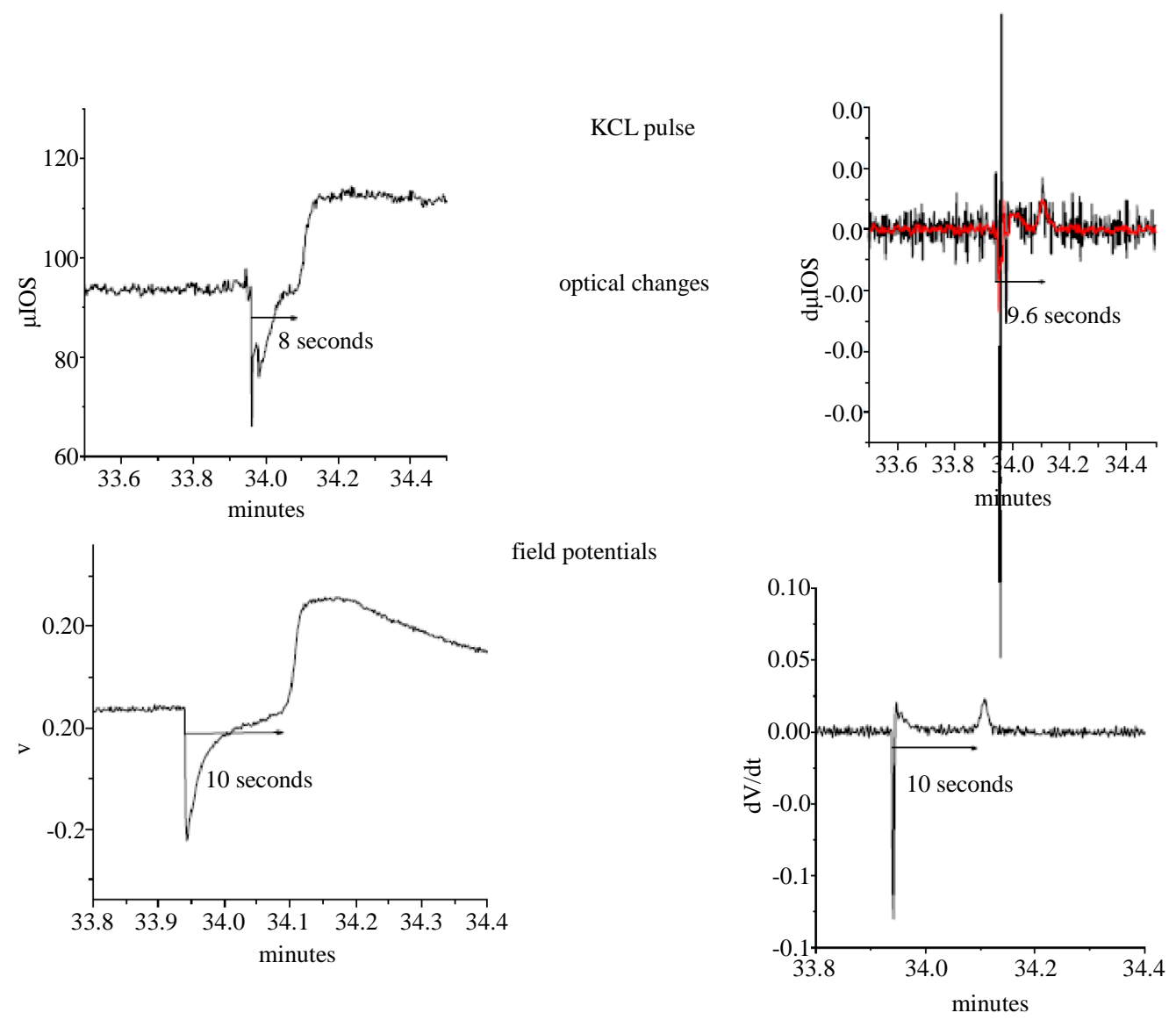

Figure 6. Same experiment as shown in Figure 6. The $\mu$ IOS and field potential time series are shown in an expanded time scale in order to highlight the details of the optical and field potential responses to the first $\mathrm{KCl}$ pulse. Top row: the optical signal shows that the hyper-transparency exceeds the artifact and precedes the light scatter, which is the hallmark of propagation. The time derivative for the time series is shown on the right hand side. Note that the stimulus artifact influences the first part of this derivative but not the peak at 9.6 seconds. Bottom row: field potential simultaneously recorded. Note the two components: a potential rise followed 10 seconds later by a potential drop that is also the hallmark of propagation. The time derivative also has two components and the second is closely associated with the second component of the $\mu$ IOS. 

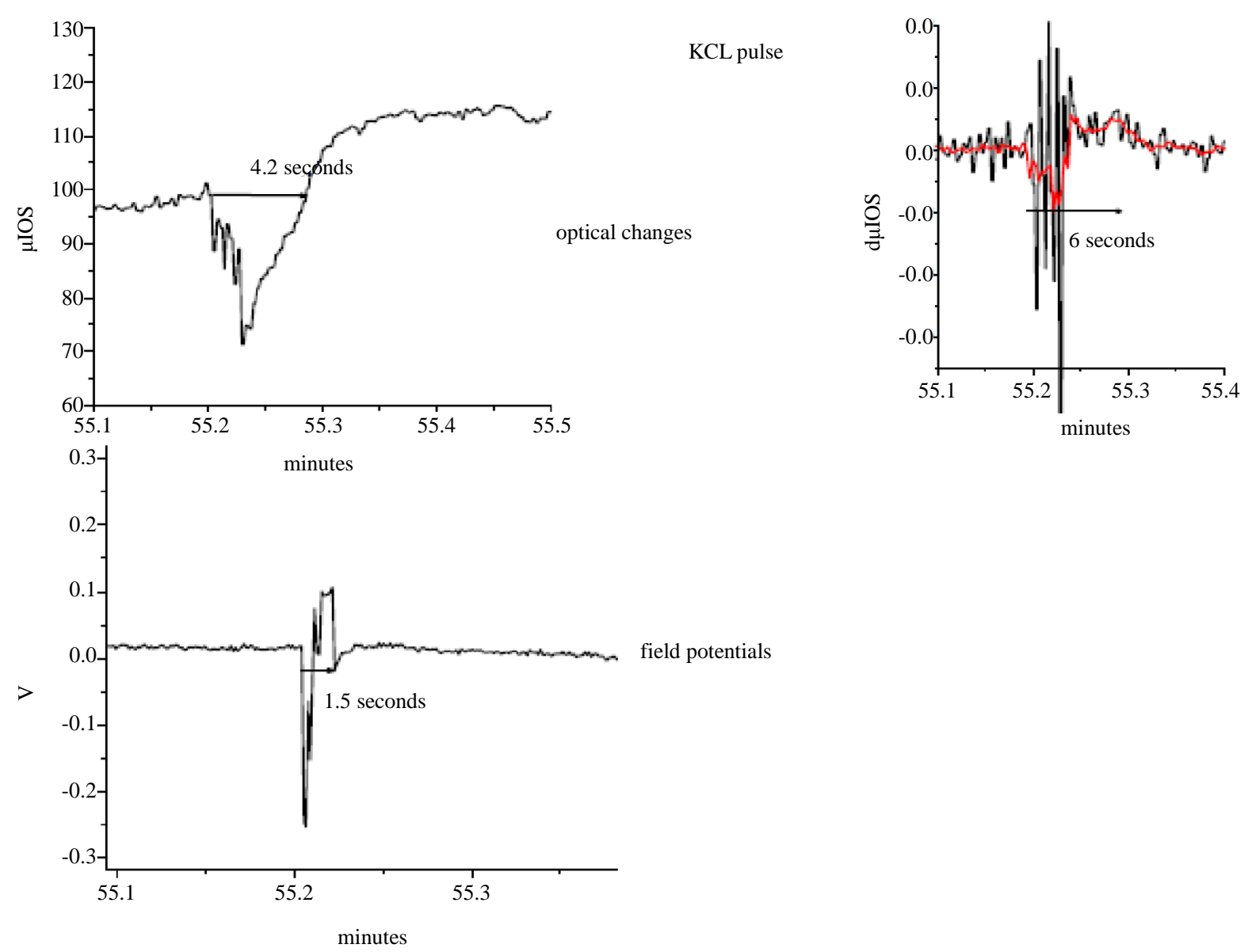

Figure 7. Same experiment as shown in Figure 6. The $\mu$ IOS and field potential time series are shown in an expanded time scale in to highlight the details of the optical and field potential responses to the second $\mathrm{KCl}$ pulse. Top row: the optical signal shows that hyper-transparency and light scatter overlap with a less defined peak after the initial response. Bottom row: the field potential has only the initial potential rise and no component associated with light scatter increase. The duration of the field potential response is the duration of the pulse. No artifact from turbulence can be seen in the field potential time series.

The stimulation artifact in the optical signal provides information about the relative durations of the stimulus and its optical response. There are two clear components in both, the electrical and optical responses, and the second component of both derivatives happen together. In the optical response to the second pulse, the two optical components overlap and there is a small second component in the time derivative. The field potential is very short and dominated by the initial potential rise. No second component was found in the time derivative. The sharp field potential rise was recorded in five of the seven pulses and absent in two. The plateau shape of the KCl-elicited wave was also recorded in five of the seven pulses.

Figure 8 shows a digital frame of the recorded DVD movie of another two-pulse experiment, which was used to construct the kymographs shown below. The color frames were first transformed into gray levels, as shown. The glass electrode and its shadow are visible in the frame. The structure to the left of the electrode is a dynamic electrochemical structure called a "standing pattern". From this structure, excitation waves spread up to the electrode tip region that is concealed by the superposition of the A/D converter output (a movie of this experiment is available as additional material for the electronic version). The asterisk indicates a small lesion that developed around the original electrode insertion point. The lesion formed after the first $\mathrm{KCl}$ pulse whereby a standing pattern arose around the insertion point, the outline of which can still be seen in the frame. When such a lesion develops, the electrode has to be moved because it becomes isolated from the electric field of approaching waves. The three lines labeled a, b, and c cut the standing pattern at different levels and these lines were used to create the kymographs shown below the frame. The alignment of the kymographs very clearly 

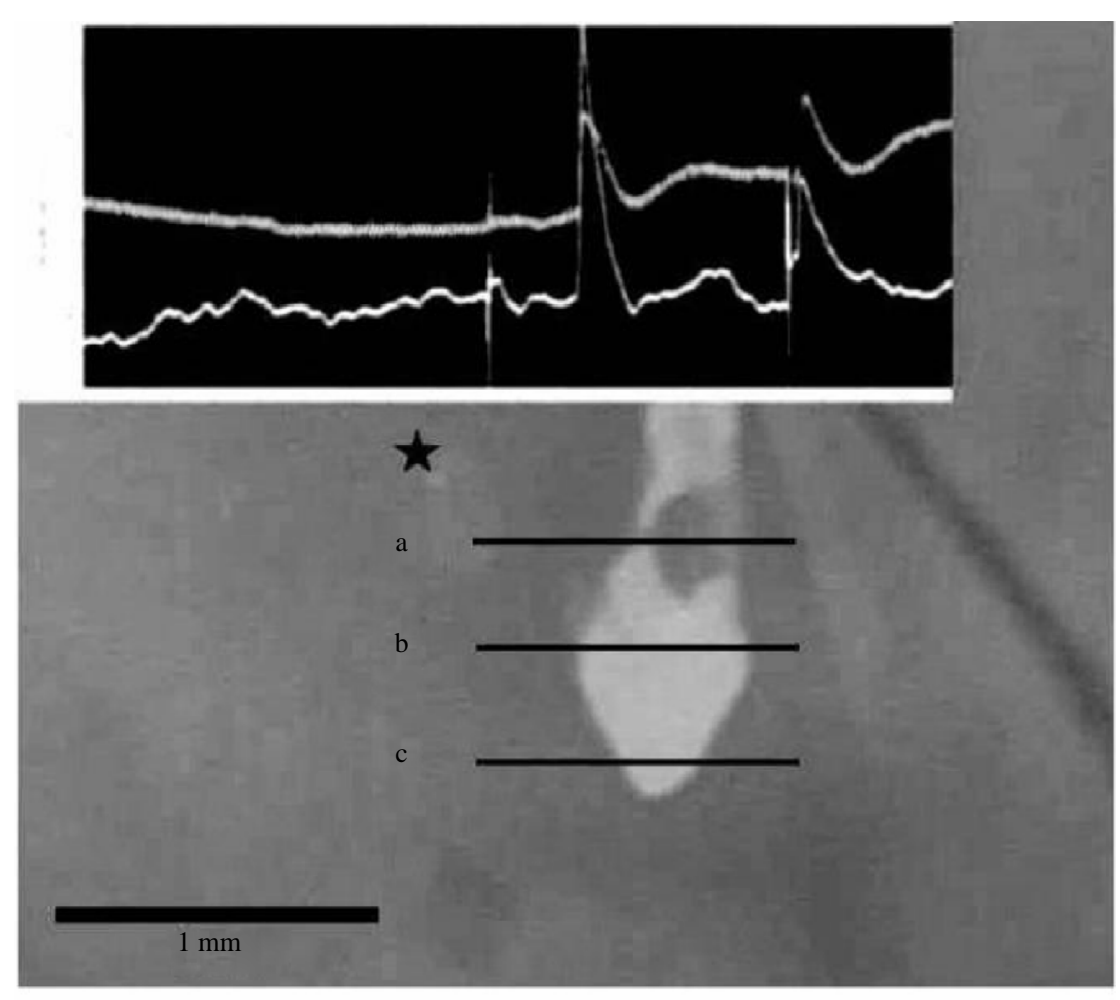

a

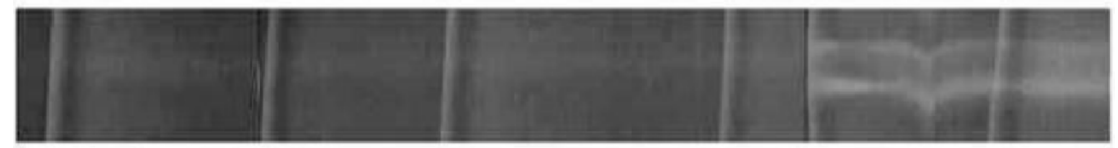

b

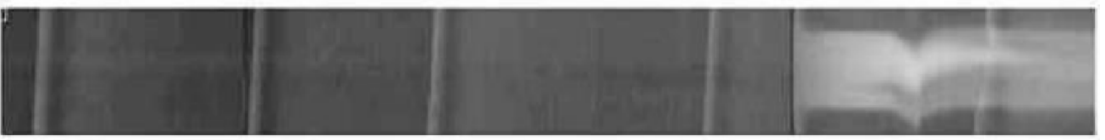

c

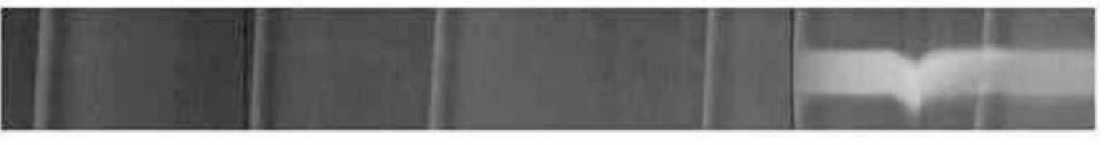

SD

KCL

SD

SD

$\mathrm{KCl}$

SD

$10 \mathrm{~min}$

Figure 8. The figure summarizes the findings of another two-KCl pulse experiment from a different retina where hyper-transparency was the first optical tissue response. The digitized frame shows the superimposed time series of the $\mu$ IOS (top) and the field potential (bottom) of the going on record. The original color frame has been transformed into grayscale at 8 bits. The bright structure in the frame is a standing pattern that arose after the second $\mathrm{KCl}$ pulse, from which the excitation propagated. The electrode shadow is better seen than the glass electrode itself. The tip is behind the superimposed time series. The asterisk indicates the location of a small bright spot that is a micro lesion that developed around the electrode tip from an earlier electrode insertion. The electrode was therefore moved from the center of the frame and its tip is behind the superimposed time series in the image. These micro lesions will always split RSD wave fronts (see additional material). Three lines labeled a, b and c cut the standing pattern at different levels. Each line was used for the stacking of the kymographs shown below. The kymograph is a digital processing technique that is used for the visualization of movement, vibrations, or changes over time. The brightness of the pixels in each line is positioned side by side over time, such that the length of the total stack equals the 62 minutes of the recorded experiment. 3442 lines were stacked with a sampling rate of $2 \mathrm{~Hz}$. The three aligned stacks then show the optical changes at three different levels of central retina. From the stacks it is apparent that hyper-transparency appears simultaneously in all three lines following both $\mathrm{KCl}$ pulses, demonstrating how fast the tissue responds to the pulses over a wide area. The evolution of the dynamic standing pattern can also be followed. Brightness does stay static within the pattern, but it changes. Note the RSD wave's propagation within each line and the interaction of a RSD wave with the standing pattern that is different at each level. 
shows the difference in spread of the circular RSD waves and the $\mathrm{KCl}$ elicited waves: the circular wave front reaches each line at different times, whereas the optical $\mathrm{KCl}$ initial pulse response is synchronous over the central retina (about $3 \mathrm{~mm}$ ). As in the experiment shown in Figures 5-7, the initial optical response is an increase in tissue transparency observed in both $\mathrm{KCl}$ pulses.

In Figure 9, the optical and field potential responses are shown in highly expanded time scale in order to reveal the details of their temporal evolution. The optical and electrical artifacts caused by the turbulence created by the pulses clearly interrupted the optical response and changed the second pulse field potential. It is obvious that during the second pulse, the electrode tip moved within the tissue and affected the initial response, which made amplitude comparisons difficult.

However, both field potential responses are qualitatively similar and in these two pulses, the initial rise in field potential was absent. The non-linearity between these macroscopic concomitants is also demonstrated in the exogenous $\mathrm{KCl}$ experiments. The kymograph also shows the fluctuation in brightness of the standing pattern and the complex interaction of the pattern with a propagating RSD. In this experiment both standing patterns disappeared without leaving lesions. Indeed, the field potential and potassium activity within such a pattern has previously been reported [4] along with the spectral dependence of its IOS [17]. From these experiments we

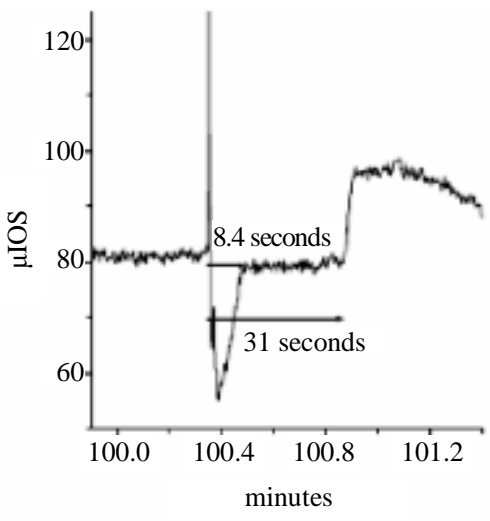

$100 \mu \mathrm{l}$

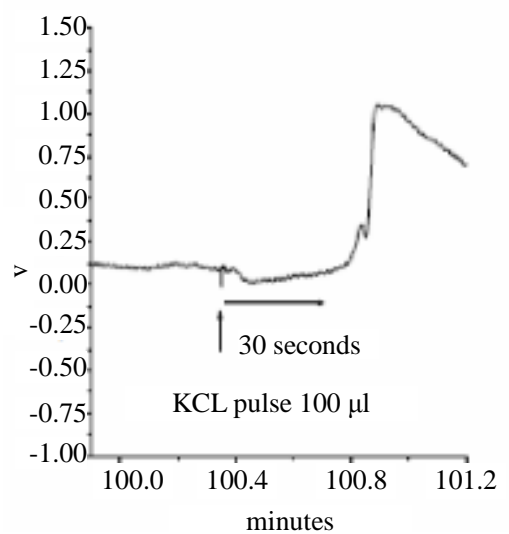

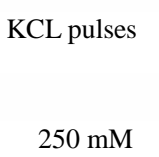

optical changes

field potentials

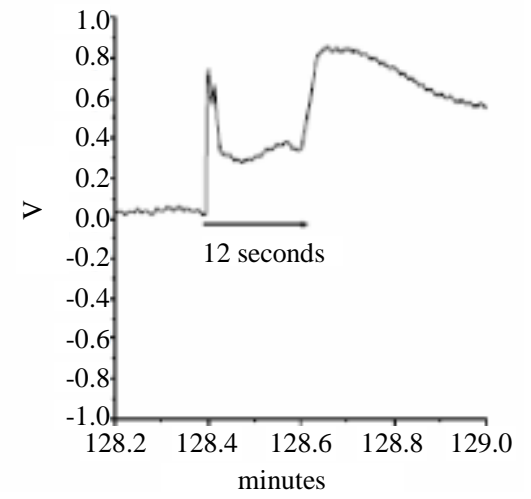

Figure 9. The graphs in this figure are from the same experiment shown in Figure 9. The time series recorded for the two $\mathrm{KCl}$ pulses are shown in expanded time scale. The first and second $\mathrm{KCl}$ pulses were $100 \mu \mathrm{l}$ and $200 \mu \mathrm{l}$ in volume, respectively. Both of the $\mathrm{KCl}$ pulses produced optical and mechanical artifacts that lasted for 2 and 3.5 seconds, respectively. The mechanical disturbance is seen at the field potential time series due to the turbulent flow of the pulse, with a stronger effect caused by the second pulse. Top row: $\mu$ IOS with two optical components well-separated in time. Bottom row: field potential time series. After the very small artifact, the field potential baseline shifted very slightly toward a positive direction before a slow ramp became faster before the main potential drop that coincided with an increase in tissue light scatter around the electrode tip. In the second pulse, the mechanical artifact of the pulse displaced the baseline, which is perhaps the reason for the lack of field rise associated with other pulses. 
conclude that the optical profiles of $\mathrm{KCl}$ pulse excitation waves can have an optical profile distinct from RSD waves. All the optical changes associated with increased extracellular potassium activity were also observed, but not in all pulses or retinas. When present, the hyper-transparency phase preceded the increase in light scatter. Finally, the field potentials frequently showed a potential rise along with two components in its time derivative, but not in all pulses.

\subsection{The Light Scatter Signals in "in Situ" Retinas Viewed from the Side}

In the methods section we describe the experimental setup used to record the IOS across the inner retina. In Figure 10 this arrangement is summarized. Two cameras were adapted to two microscopes: one camera had a panoramic view of the retina from above, while the second was focused on the side view of a patch of central retina illuminated by a red laser beam. The images of the two cameras were combined into a single frame and recorded together, and then processed offline.

Figure 11 shows a sequence of frames that begin one second before a RSD wave invades the area, and then follow the optical changes in the transition from quiescence to excitation wave.

In part E of Figure 10 we show the processed frame together with the drawings of Ramon y Cajal of Müller cells in the chicken retina. The characteristic circular patterns of the glial membrane around the ganglion cell bodies are visible in both figures. Below is the inner plexiform layer, which occupies one third of the retina width. Above the ganglion cells is the nerve fibers layer that converges toward the optic nerve papilla. The endfeet of the Müller cells form the border between the retina and basement membrane of the inner-limiting membrane. To construct the frames shown in Figure 10 and Figure 11, a sequence of 300 frames was digitized from the original video, beginning one second before a front-wave invaded the area. The 300 frames represent 10 seconds of recording time (i.e., the rising phase of the field potential). The frames were saved in grayscale (8 bits) and the first frame used as the background, i.e., the value of the brightness of every pixel in this frame was subtracted for all subsequent 299 frames. Then we employed a false colordisplay. In part F of Figure 11, the mean brightness of three small rectangular matrices is displayed as time series. We measured the mean brightness only in the inner retina in order to compare the behavior of the optical changes within the plexiform and both nuclear layers. At the inner plexiform layer, the brightness changes within the second before the wave and has an abrupt step at the transition from quiescence to excitation ( 100 msec). We used red laser light because the scatter of red light matches the membrane depolarization obtained with the voltage-sensitive dye 4ANEPPS [4] [34] and thus closely follows the field potential of a wave [17].

\section{Discussion}

In this section we will argue for: 1) a prominent role of intrinsic and membrane-associated polyelectrolytes (polyanions) in the generation of the field potential recorded at the inner plexiform layer; and 2) a prominent role of the glial membrane network in the generation of the IOS of dynamic structures in in vitro retinas. We found that glial membrane channel activity measured in situ had the same shape and duration of the rise of the extracellular field potential [6]. Intracellular, the glia light-flash response and RSD wave responses were very similar, suggesting that the b-wave of the ERG and field potential drop of RSD waves shared the same mechanism of glial membrane depolarization [7]-[9] [35]. By contrast, ganglion cells had a very short burst of activity at wave onset and the response of horizontal cells to light appeared to be unaffected by RSDs (idem).

Being inserted in the extracellular space of the inner plexiform layer, the electrode tip is surrounded by polyanions of the glycosaminoglycans (GAGs) that are associated with glia and synaptic membranes, as well as negatively charged glycolipids (gangliosides), glycoproteins, and proteoglycans that are intrinsic to these membranes. These polyelectrolytes react to electrical fields and create interfacial potentials when the screening of the negative charges is changed. Typically, polyanions undergo marked volume changes when hydrated. Mucins (the largest of the proteoglycans) for example, can experience a 600 -fold change in volume within seconds after exocytosis. Such a change happens in goblet cells when protons and calcium ions screen the very large and tightly folded mucin macromolecule [36], and the fast hydration of the resultant negatively charged carbohydrates is responsible for the volume changes [37]. Thesevolume changes have been described as "volume phase transitions” by T. Tanaka (1992) [38].

Other scientists measured field potential drops in hydrated hydrogels [39] and monitored the effect of different $\mathrm{KCl}$ solutions ( $0.8 \mathrm{mM}$ and $5 \mathrm{M}$ ) and pHs. In the presence of low concentration $\mathrm{KCl}$ solutions, they observed 


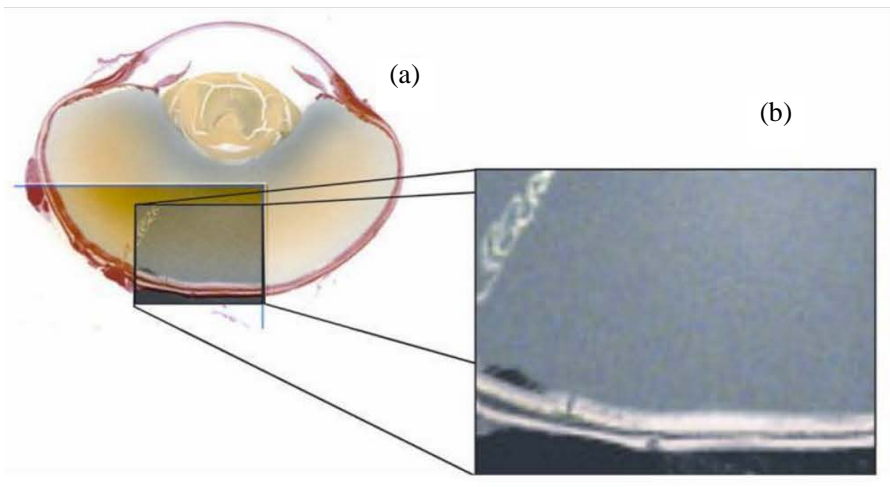

(c)
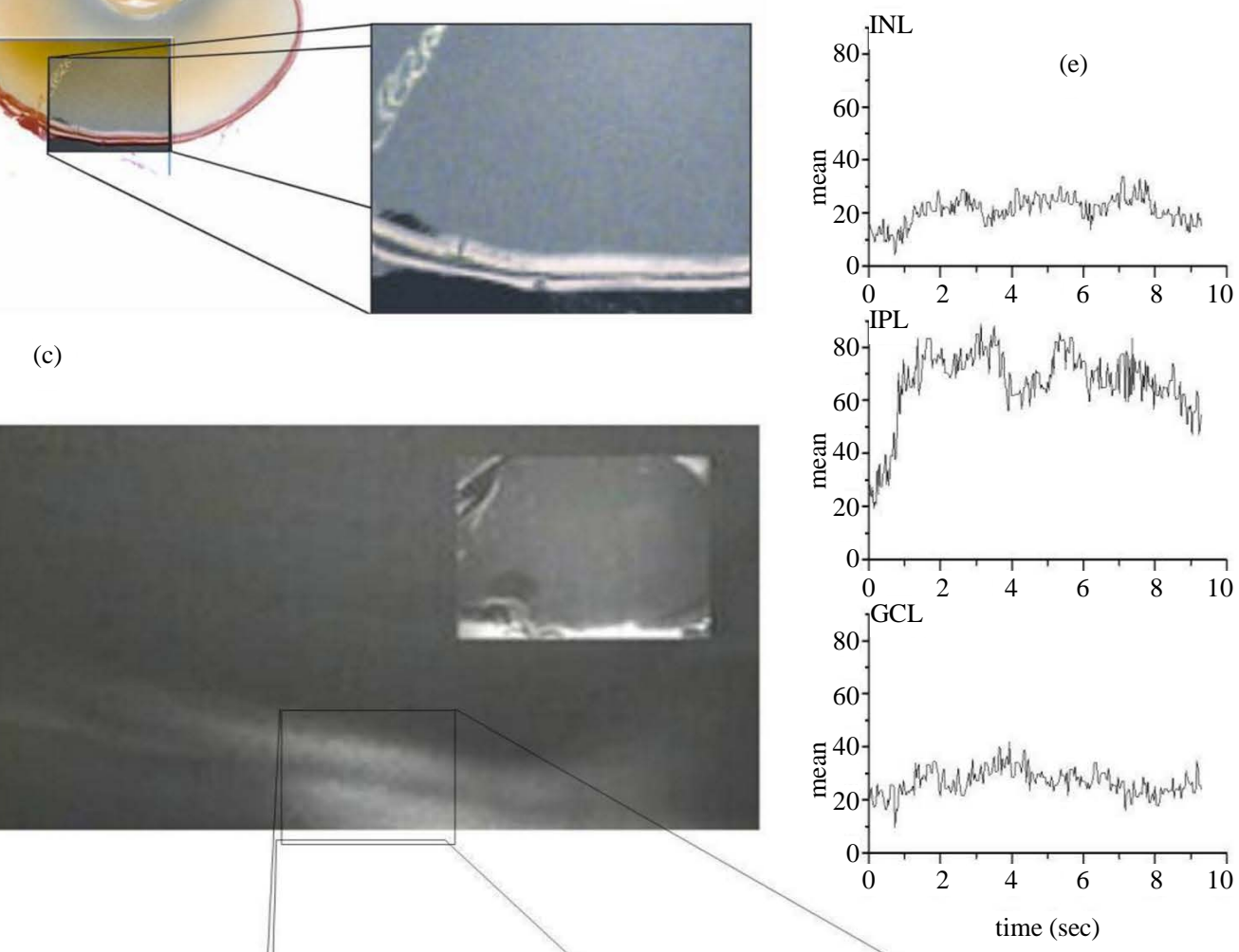

(d)

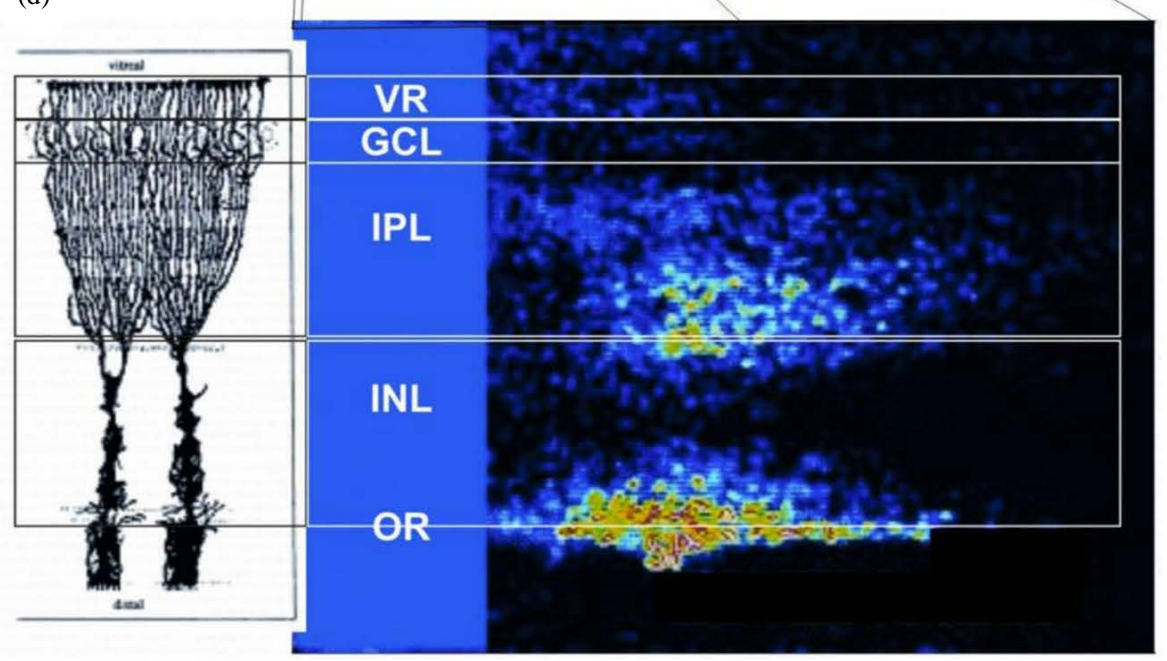

(e)

Figure 10. (a) A chicken eye with the boxed area shown in more detail in (c). (b) Part of the retinal tissue can be seen from above and additionally the retina can be seen from the side, from which the layer structure can be recorded. Part (c) shows this in more detail. The given frame has the side view at high resolution, and the inset shows the complete retina from above. Part (d) of the figure shows a reproduction of an old drawing from Cajal of Mueller cells to highlight their global structure. Part (e) shows a side view under laser illumination in false colors at the time a wave passes. Part (f) shows the brightness of different layers in time at the passage of a wave front. Whereas the IPL has a significant sudden jump at the wave front, the signal changes in the INL and GCL are, when present at all, small. According to part (d) of this figure see: Cajal S. R. In: Thorpe S. A., Glickstein M., translators. 1892. The structure of the retina. Springfield (IL): Thomas, 1972 [40]. 


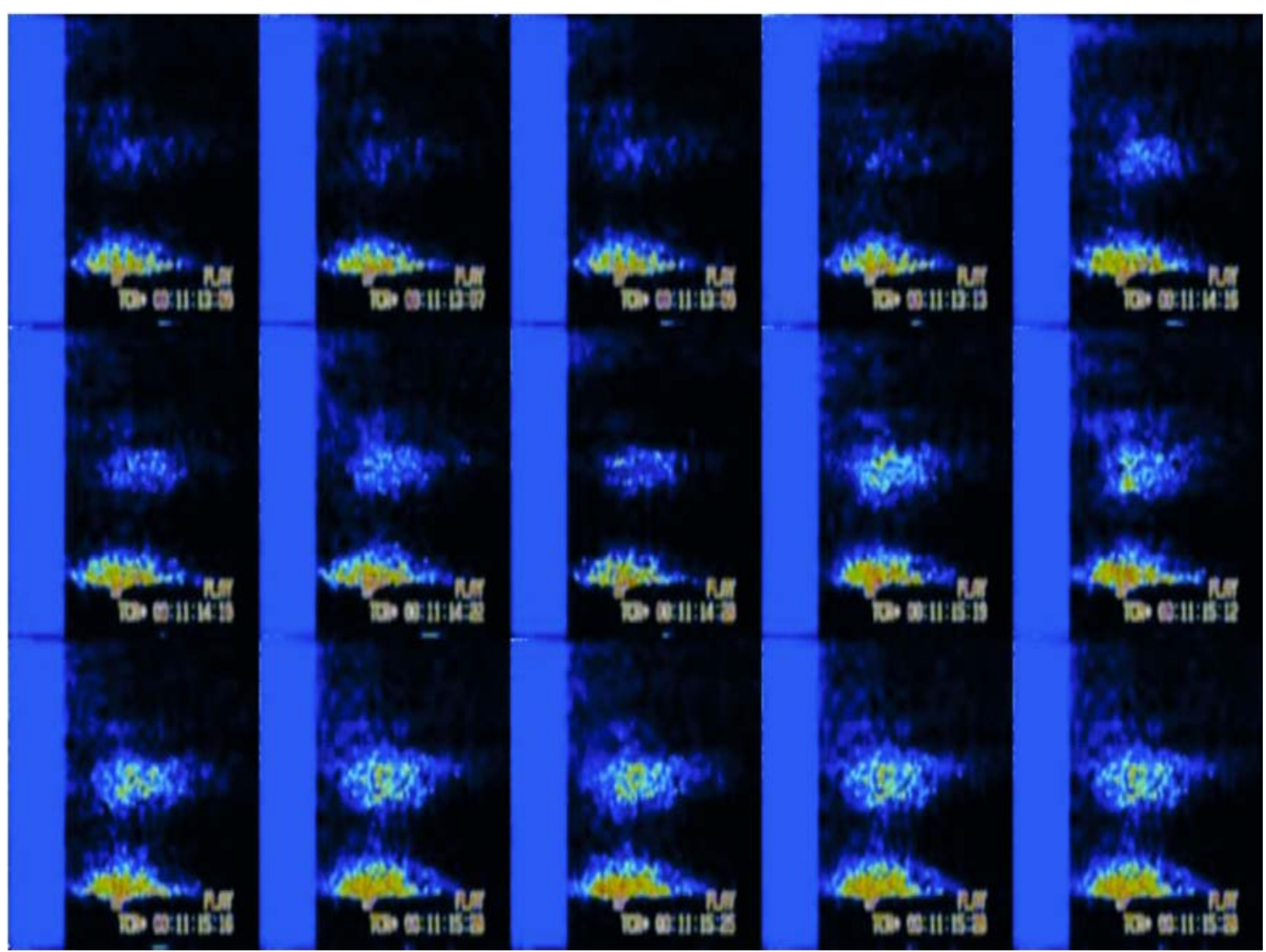

Figure 11. Sequence of frames showing a retina while a spreading depression front is passing, illuminated from the side by a red laser. The construction of these frames and the layer structure of the retina are shown in Figure 10. The IPL first and almost exclusively shows an optical change as a consequence of the incoming wave.

potentials of $120 \mathrm{mV}$, while in high concentrations, the potential dropped to about half of that value. Additionally, it was found that the field potential of the hydrogels was much smaller at $\mathrm{pH} 3$ than at $\mathrm{pH}$ 7. This demonstrates that the anionic hydrogel possesses significant negative potential in salt solutions.

It is known that fast volume transitions of biological polyanions can be completely reversible, as shown by the expansion and contraction of heparin-sulfate due to $\mathrm{pH}$, and of histamine after exocytosis from mast cells [41]. This is because when a wave approaches a patch of the inner plexiform layer, polyanionic macromolecular complexes undergo quaternary structural changes that in turn add to the field potential. There is therefore a tight coupling between field potential and optical changes, since light scatter is influenced by geometry.

Two additional pieces of experimental evidence suggest mechano-electrical coupling in RSD waves: 1) the mechanical displacement of retinas [42] at wave onset; and 2) liberation of heat with further absorption [43] that is compatible with relaxation then recovery of structure. In electrochemical systems, the field potential is important for long-range correlations within the system. In our experimental model, it appears that these longrange correlations depend on the integrity of the inner-limiting membrane. Retinas were cut horizontally such that the inner retina was separated from the inner nuclear layer and outer retina [42]. In these reduced preparations, RSD waves could be recorded for hours with electrical and optical signals identical to those recorded from intact retinas. By contrast, it has previously been shown that the thinnest cut made at the inner limiting membrane stops the waves [44]. More recently, we observed the collapse of retinal excitability in the presence of protamine that had been added to the maintenance solution in nanomolar concentration [45]. At $22 \mathrm{nM}$, the collapse was complete in three minutes and persisted for hours. At the same time, the tissue transparency increased. In retinas transparency and physiological state are synonymous, that is, in order to be functional, retinas must be transparent. It then follows that protamine rendered the retinas unexcitable without any sign of toxicity. In a re- 
lated example, protamine blocks transport in epithelium without interfering with the tissue potential (i.e. the cells maintain polarity) [46] [47]. In axons, protamine also induces collapse of excitability without change in the membrane potential. Since this cationic peptide makes stable heteropolymers with heparin in plasma [48], the polymerization of the polyanions at the inner limiting membrane could explain the protamine effect on retinal excitability. Such an effect is a glia response, since only the endfeet of glia are present at the inner limiting membrane. It should be noted that in the chicken retina, Müller cells have no gap junctions [4] [49]. At the epithelium, it has also been observed that protamine changes the structure of the basement membrane while sparing the structure of the gap junction [46] [47].

The detection of circular waves where the relatively large optic nerve papilla does not interfere with the pattern clearly indicates that the radial glia and the extracellular matrix are sufficient to maintain propagation. In these two dimensional patterns, curvature and velocity are related [24]. While the lack of cell bodies or synaptic terminals does not affect propagation, micro lesions will always split the waves. In Figure 9 the asterisk shows a small dot that is a micro lesion that appeared around the microelectrode. When this happens, the tissue loses transparency around the electrode and the electrode is isolated from the field potential of approaching waves. This indicates that there is a break in long-range correlations cause by the small lesion that splits the wave front in two (not shown). The split of waves by micro lesions and the lack of effect of the optical papilla on RSD wave propagation has been noted literally hundreds of times, for example see [50] and the first report on RSD waves in chick retina [25].

When a wave invades a patch of retina, several electrochemical concomitants are recorded in the first 10 seconds: the field potential drop, an abrupt rise in potassium activity, and a $\mathrm{pH}$ shift in the alkaline direction. At the peak of dK/dt, calcium, sodium and chloride activities fall. All these changes in ion activity have been interpreted in terms of transmembrane movements through channels. The fact that ion activity also falls or rises depending on the screening of countercharges is rarely discussed. It is possible that both mechanisms play a part in ion activity changes. The interaction between ions and polyelectrolytes is called the lyotropic effect and ions with the same charge can have different effects [51] [52]. For instance, the chaotropic potassium and organotropic sodium exert different effects on polyanions. The same is true for calcium and magnesium [53].

Membranes are lipid bilayers with protein and sugar complexes either integrated or associated. Short pulses of ion solutions change the surface potential of lipid bilayers [54]. The short $\mathrm{KCl}$ pulses would then change the endfeet surface potential. If we take an estimate of 15,000 glial cells per $\mathrm{mm}^{2}$ and about 12 endfeet per cell [4] [14], we arrive at 180,000 endfeet per $\mathrm{mm}^{2}$. The synchronous hyper-transparency that we observed with four $\mathrm{KCl}$ pulses can be explained by a glia membrane mass response to the sudden increase in surface potential. Glia depolarization, on the other hand, is the main contributor to light scatter. The relationship between membrane potential and the IOS is linear for neurons in culture. In this experimental situation the contribution to the IOS was much larger in the fine axon sprouts and very small in the cell bodies. The linear relationship was measured over the physiological range of potential $(\Delta \mathrm{S} / \mathrm{S}$ depended linearly on $\Delta \mathrm{V} / \mathrm{V}$ in the range $-100 \mathrm{mV}$ to $100 \mathrm{mV})$. What is lacking in the culture dish is the extracellular matrix gel. Thus this finding is also compatible with the non-linearity measured in intact tissue due to the gel's non-linear response to $\mathrm{pH}$ and ionic environment. Potassium activity increases the potential of bilayers [54] and at the same time decreases the interfacial potential of polyanionic gels [40]. Depending on what effect predominates around the electrode tip, a rise or fall in field potential can be measured.

All the electrochemical wave concomitants have a maximum at the IPL [11] [55] and in the experiment shown in Figure 10 and Figure 11, we saw that the IOS associated with RSD is also maximal at the IPL. Using a laser confocal microscope, Reichenbach and Bringmann (2010) [14] showed that light scattering is strong in the two plexiform layers of freshly isolated retinas, being strongest at the IPL in the scotopic retina of the guinea pig. The same observation has been made by Franze et al. (2007) [15]. Light scatter is maximal at IPL in live retinas. Using red laser light we replicated the findings of Martins-Ferreira [56] on terms of the origin of the IOS associated with RSD. The increase in light scatter at the IPL preceded the wave front and was maximal at this layer, yet the absence of synaptic terminals at the optic nerve papilla does not affect the IOS or propagation velocity of solitary waves. Thus it is fair to conclude that the glial network and matrix are sufficient for IOS generation.

The other feature observed with the exogenous potassium pulses was the shape of the optical profiles. After an initial fast recovery, the optical signal falls to a plateau with slow recovery. This pattern was present in the response to five of the seven pulses in four of the five tested retinas. The simplest explanation for this shape is 
that the two components observed with circular wave fronts are fused in the profile of the $\mathrm{KCl}$ response. The second component of the RSD profiles is closely associated with lactate secretion by glia and a slow pH shift in the acid direction at the extracellular space [29]. The metabolism of the avascular chicken retina in vitro is $100 \%$ dependent on glycolysis driven by the glial sodium pump [14] [21] [57]. This pump in turn is driven by extracellular potassium activity [58], unlike the pumps of neurons and muscle that are driven by intracellular sodium activity [59]. As is the case of the muscle membrane pump, the behavior of the glial pump is adaptive [58]. The maximum rate is reached at $12 \mathrm{mEq} / \mathrm{l}$ potassium (idem). Regardless of whether this explanation is the correct one, the fact is that responses to $\mathrm{KCl}$ pulses can generate a different profile from circular RSD waves elicited mechanically or electrically. In recent papers we have shown that excitotoxic responses also differ in optical profiles from circular wave fronts [21]. Thus, optical profiles can be used in the classification of excitation states of tissue.

\section{Conclusion}

In conclusion, the RSD waves and other electrochemical patterns observed in isolated retinas provide a widow to the non-linear neural-glial dynamics of all gray matter. If the excitable media theory context is applied to these patterns, then results from the retina can be extended to cortex and heart tissue provided that geometrical and metabolic considerations are taken into account. The probabilities of solitary wave fronts, standing patterns, and self-sustained sequences of waves will be different in each tissue but the rules for emergence and propagation will be similar in the one spatial dimension of action potentials, two dimensions of RSD waves in retina, and more complex three dimensions of cortical SDs and each heartbeat. The non-linear behavior of polyanionic gels, for example, will very likely play an important role in all electrochemical patterns of excitable tissue. The membrane model that is explicit about the role of polyanionic gels in explaining mechanical, optical, and thermal events concomitant with excitation is the Ichigi Tasaki membrane model [60].

\section{Acknowledgements}

The authors wish to dedicate this paper to the memory of Ichigi Tasaki and his membrane model.

\section{References}

[1] Zykov, V. (2008) Excitable Media. Scholarpedia, 3, 1834. http://dx.doi.org/10.4249/scholarpedia.1834

[2] Flatgen, G., Krischer, K., Pettinger, B., Doblhofer, K., Junkes, H. and Ertl, G. (1995) Two-Dimensional Imaging of Potential Waves in Electrochemical Systems by Surface Plasmon Microscopy. Science, 269, 668-671. http://dx.doi.org/10.1126/science.269.5224.668

[3] Fernandes de Lima, V.M., Scheller, D., Tegtmeyer, F., Hanke, W. and Schlue, R. (1993) Self-Sustained Spreading Depression in the Chicken Retina and Short Term Neuronal-Glial Interactions within the Gray Matter Neuropil. Brain Research, 614, 45-51. http://dx.doi.org/10.1016/0006-8993(93)91016-L

[4] Fernandes de Lima, V.M. and Hanke, W. (1997) Excitation Waves in Central Grey Matter: The Retinal Spreading Depression. Progress in Retinal and Eye Research, 6, 657-690. http://dx.doi.org/10.1016/S1350-9462(96)00038-9

[5] Dahlem, M.A. and Hadjikhani, N. (2009) Migraine Aura: Retracting Particle-Like Waves in Weakly Susceptible Cortex. PLOSone, 4, 1-10. http://dx.doi.org/10.1371/journal.pone.0005007

[6] Hanke, W., Fernandes de Lima, V.M. and Schlue, W.-R. (1993) Patch-Clamp Experiments in the Intact Chicken Retina during Spreading Depression. In: Lehmenkühler, A., Grotemeyer, K.H. and Tegtmeyer, F., Eds., Migraine: Basic Mechanisms and Treatment, Urban \& Schwarzenberg, Munich.

[7] Mori, S., Miller, W.H. and Tomita, T. (1976) Microelectrodes Study of Spreading Depression (SD) in the Frog Retina. General Observation of Field Potentials Associated with SDs. The Japanese Journal of Physiology, 26, 203-217. http://dx.doi.org/10.2170/jiphysiol.26.203

[8] Mori, S., Miller, W.H. and Tomita, T. (1976) Microelectrode Study of Spreading Depression (SD) in Retina Müller Cell Activity and $\left[\mathrm{K}^{+}\right]$during SD. The Japanese Journal of Physiology, 26, 219-233. http://dx.doi.org/10.2170/jiphysiol.26.219

[9] Mori, S., Miller, W.H. and Tomita, T. (1976) Müller Cell Function during Spreading Depression in the Frog Retina. PNAS, 73, 1351-1354. http://dx.doi.org/10.1073/pnas.73.4.1351

[10] Harreveld, A.V. and Fifkova, E. (1972) Effects of Metabolic Inhibitors on the Release of Glutamate from the Retina. Journal of Neurochemistry, 19, 1439-1450. http://dx.doi.org/10.1111/j.1471-4159.1972.tb05087.x 
[11] DoCarmo R.J. and Martins-Ferreira H. (1984) Spreading Depression of Leão Probed with Ion-Selective Microelectrodes. Anais da Academia Brasileira de Ciências, 56, 401-421.

[12] Dahlem, Y.A., Dahlem, M., Mair, T., Braun, K. and Mueller, S.C. (2003) Extracellular Potassium Alters Frequency and Profile of Retinal Spreading Depression. Experimental Brain Research, 152, 221-228. http://dx.doi.org/10.1007/s00221-003-1545-y

[13] Fernandes de Lima, V.M., Piqueira, J.R.C. and Hanke, W. (2009) The Synergetic Modulation of the Excitability of Central Gray Matter by a Neuropeptide: Two Protocols Using Excitation Waves in the Chicken Retina. Anais da Academia Brasileira de Ciências, 81, 1-11.

[14] Reichenbach, A. and Bringmann, A. (2010) Müller Cells in the Healthy and Diseased Retina. Springer, New York, Dordrecht, Heidelberg and London. http://dx.doi.org/10.1007/978-1-4419-1672-3

[15] Franze, K., Groshe, J., Skatchkov, S.N., Schinkinger, S., Foja, C., Schild, D., Uckermann, O., Travis, K. and Reichenbach, A. (2007) Müller Cells Are Living Optical Fibers in the Vertebrate Retina. Proceedings of the National Academy of Sciences of the United States of America, 104, 8287-8292. http://dx.doi.org/10.1073/pnas.0611180104

[16] Fernandes de Lima, V.M., Kogler, J.E., Bennaton, J. and Hanke, W. (2001) Wave Onset in Central Grey Matter: Its Intrinsic Optical Signal and Phase Transitions in Extracellular Polymers. Anais da Academia Brasileira de Ciências, 73, 351-364. http://dx.doi.org/10.1590/S0001-37652001000300006

[17] Fernandes de Lima, V.M., Weimer, M. and Hanke, W. (2002) Spectral Dependence of the Intrinsic Optical Signal of Excited States of Central Gray Matter and Conformational Changes at Membrane Interfaces. Physical Chemistry Chemical Physics, 4, 1374-1379. http://dx.doi.org/10.1039/b109914k

[18] Fernandes de Lima, V.M., Spencer, P. and Hanke, W. (2014) Interaction of Small Cationic Peptides with Intact Basement Membranes. A Study Using Intrinsic Optical Signals of Chick Retinas. Current Medicinal Chemistry, 21, 14581466.

[19] Da Silva, J.A.A., Spencer, P., Camillo, M.A. and Fernandes de Lima, V.M. (2012) Gyroxin and Its Biological Activity: Effects on CNS Basement Membranes and Endothelium and Protease Activated Receptors. Current Medicinal Chemistry, 19, 281-291. http://dx.doi.org/10.2174/092986712803414123

[20] Sieber, M. (2011) Neuroproctetive Effect of Nicotine. Current Medicinal Chemistry, 2, 292-297.

[21] Fernandes de Lima, V.M. and Hanke, W. (2012) The Kinetics of Non-Synaptically Triggered Acute Excitotoxic Responses in the Central Nervous System Observed Using Intrinsic Optical Signals. CNS \& Neurological Disorders Drug Targets, 11, 132-141. http://dx.doi.org/10.2174/187152712800269704

[22] Fischer, A.J., Zelinka, C. and Scott, M.A. (2010) Heterogeneity of Glia in the Retina and Optic Nerve of Birds and Mammals. PLoS ONE, 5, e10774. http://dx.doi.org/10.1371/journal.pone.0010774

[23] Schuck, J., Gerhardt, H. and Wolburg, H. (2000) Theperipappilary Glia of the Optic Nerve Head in the Chicken Retina. The Anatomical Record, 259, 263-275. http://dx.doi.org/10.1002/1097-0185(20000701)259:3<263::AID-AR40>3.0.CO;2-W

[24] Davidov, V.A., Manz, N., Steinbock, O. and Müller, S. (2002) Critical Properties of Excitation Waves on Curved Surfaces: Curvature-Dependent Loss of Excitability. Europhysics Letters, 59, 344-350. http://dx.doi.org/10.1209/epl/i2002-00200-6

[25] Martins-Ferreira, H. and Oliveira e Castro, G. (1966) Light-Scattering Changes Accompanying Spreading Depression in Isolated Retina. Journal of Neurophysiology, 29, 239-279.

[26] Brand, S., Dahlem, M., Fernandes de Lima, V.M. and Hanke, W. (1997) Dispersion Relation of Spreading Depression Waves in the Chicken Retina. International Journal of Bifurcation and Chaos, 7, 1359-1365. http://dx.doi.org/10.1142/S0218127497001072

[27] Brand, S., Fernandes de Lima, V.M. and Hanke, W. (1998) Pharmacological Modulation of the Refractory Period of the Retinal Spreading Depression. Naunyn-Schmiedeberg's Archives of Pharmacology, 357, 419-425. http://dx.doi.org/10.1007/PL00005188

[28] Weimer, M. and Hanke, W. (2005) Correlation between the Durations of Refractory Period and Intrinsic Optical Signals of Retinal Spreading Depression during Temperature Variations. Experimental Brain Research, 161, 201-208. http://dx.doi.org/10.1007/s00221-004-2060-5

[29] Ferreira Filho, C.R. and Martins-Ferreira, H. (1992) Interstitial Fluid pH and Its Change during Spreading Depression in Isolated Chicken Retina. In: Do Carmo, R., Ed., Spreading Depression, Springer Verlag, Berlin, Heidelberg and New York, 75-88.

[30] Peixoto, N.L.V. (1997) A Depressãoalastrantena Retina. Master’s Thesis, Universidade Estadual de Campinas, Campinas, 102.

[31] Grafstein, B. (1956) Mechanism of Spreading Cortical Depression. Journal of Neurophysiology, 19, $154-171$. 
[32] Haglund, M.M. and Schwartzkroin, P.A. (1990) Role of Na-K Pump Potassium Regulation and IPSPs in Seizures and Spreading Depression in Immature Rabbit Hippocampal Slices. Journal of Neurophysiology, 63, 225-239.

[33] Fayuk, D., Aitken, P.G., Somjem, G.G. and Turner, D.A. (2002) Two Different Mechanisms Underlie Reversible, Intrinsic Optical Signals in Rat Hippocampal Slices. Journal of Neurophysiology, 87, 1924-1937.

[34] Cajal, S.R. (1972) The Structure of the Retina. In: Thorpe, S.A. and Glickstein, M., Translators. Thomas, Springfield.

[35] Fernandes de Lima, V.M., Goldermann, M. and Hanke, W. (1994) Calcium Waves in Grey Matter Are Due to Voltage Sensitive Glial Membrane Channels. Brain Research, 663, 77-83. http://dx.doi.org/10.1016/0006-8993(94)90464-2

[36] Miller, R.F. and Dowling, J.E. (1970) Intracellular Responses of the Müller (glia) Cell of Mudpuppy Retina. Their Relation to the b-Wave of the Electroretinogram. Journal of Neurophysiology, 33, 323-341.

[37] Perez-Vilar, J. and Marbolo, R. (2007) Gel Forming Mucins. Notions from in Vitro Studies. Histology and Histopathology, 22, 455-464.

[38] Kesimer, M. and Sheehan, J.K. (2008) Analyzing the Functions of Large Glycoconjugates through the Dissipative Properties of Their Absorbed Layers Using the Gel Forming Mucin MUC5B as an Example. Glycobiology, 18, 463472. http://dx.doi.org/10.1093/glycob/cwn024

[39] Tanaka, T. (1992) Phase Transitions of Gels. In: Harlan, R., Ed., Polyelectrolyt Gels, American Chemical Society, Washington DC, 1-21.

[40] Gao, F., Reitz, F.B. and Pollack, G. (2003) Potentials in Anionic Polyelectrolytes Hydrogels. Journal of Applied Polymer Science, 59, 1319-1321. http://dx.doi.org/10.1002/app.12283

[41] Fernandez, J.M., Villalon, M. and Verdugo, P. (1991) Reversible Condensation of Mast Cell Secretory Products in Vitro. Biophysical Journal, 59, 1022-1027. http://dx.doi.org/10.1016/S0006-3495(91)82317-7

[42] Oliveira e Castro, G. and Martins-Ferreira, H. (1971) Deformations and Thickness Variations Accompanying Spreading Depression in the Retina. Journal of Neurophysiology, 33, 891-900.

[43] Tasaki, I. and Byrne, P.M. (1992) Heat Production Associated with Spreading Depression in the Amphibian Retina. Biochemical and Biophysical Research Communications, 174, 293-297. http://dx.doi.org/10.1016/0006-291X(91)90519-D

[44] Goldermann, M. and Hanke, W. (1998) Long Distance Interaction in Excitation-Depression Events of the Central Nervous System. Journal of Brain Research, 37, 210.

[45] Fernandes de Lima, V.M. and Hanke, W. (2014) Relevance of Excitable Media Theory and Retinal Spreading Depression Experiments in Preclinical Pharmacological Research. Current Neuropharmacology, 12. http://eurekaselect.com/123047

[46] Alavi, N., Lianos, E., Andres, G. and Bentzel, C.J. (1982) Effect of Protamine on the Permeability and Structure of Rat Peritoneum. Kidney International, 21, 44-53. http://dx.doi.org/10.1038/ki.1982.7

[47] Bentzel, C.J., Fromm, M., Palant, C.E. and Hegel, U.J. (1987) Protamine Alters Structure and Conductance of Necturus Gallbladder Tight Junctions without Major Electrical Effects on the Apical Cell Membrane. The Journal of Membrane Biology, 95, 9-20. http://dx.doi.org/10.1007/BF01869626

[48] Maurer, J., Haselbach, S., Klein, O., Baykut, D., Vogel, V. and Mantelle, W. (2011) Analysis of the Complex Formation of Heparin with Protamine by Light Scattering and Analytical Centrifugation Implications for Blood Coagulation Management. Journal of the American Chemical Society, 133, 1134-1140. http://dx.doi.org/10.1021/ja109699s

[49] Wiedemann, M. and Hanke, W. (1997) The Chicken Retina as a Model for Investigation of Central Nervous System Lesions. Neuroscience Letters, 232, 99-102. http://dx.doi.org/10.1016/S0304-3940(97)00578-8

[50] Collins, K.D. (2004) Ions from the Hofmeister Series and Osmolytes: Effects on Proteins in Solution and in the Crystallization Process. Methods, 34, 300-311. http://dx.doi.org/10.1016/j.ymeth.2004.03.021

[51] Zhang, Y. and Cremer, P.S. (2006) Interactions between Macromolecules and Ions: The Hofmeister Series. Current Opinion in Chemical Biology, 10, 658-663. http://dx.doi.org/10.1016/j.cbpa.2006.09.020

[52] Williams, R.J.P. (1970) Tilden Lecture. The Biochemistry of Sodium, Potassium, Magnesium and Calcium. Quarterly Reviews, Chemical Society, 24, 331-365. http://dx.doi.org/10.1039/qr9702400331

[53] Ladewig, T., Hanke, W., Guimarães de Almeida, A.C. and Fernandes de Lima, V.M. (1998) Distribution of Gap Junctions in the Chicken Retina. Journal für Hirnforschung, 39, 77-86.

[54] Laver, D.R. and Curtis, B.A. (1996) Surface Potentials Measure Ion Concentrations near Lipid Bilayers during Rapid Solution Changes. Biophysical Journal, 71, 722-731. http://dx.doi.org/10.1016/S0006-3495(96)79271-8

[55] Martins-Ferreira, H. and Do Carmo, R.J. (1987) Retinal Spreading Depression and the Extra-Cellular Milieu. Canadian Journal of Physiology and Pharmacology, 65, 1092-1110. http://dx.doi.org/10.1139/y87-171

[56] Martins-Ferreira, H. and Oliveira e Castro, G. (1971) Spreading Depression in Isolated Chick Retina. Vision Research, 
3, 171-174. http://dx.doi.org/10.1016/0042-6989(71)90038-1

[57] Fernandes de Lima, V.M., Scheller, D., Hanke, W. and Schlue, W.-R. (1993) Neuronal-Glial Interactions during "En Masse” Propagating Activity: The Retinal Spreading Depression as a Good Pharmacological Tool. In: Lehmemkueller, A., Grotemeyer, K.H. and Tegtmeyer, F., Eds., Migraine: Basic Mechanisms and Treatment, Urban-Schwarzenberg, Munich, Vienna and Baltimore, 547-562.

[58] Reichenbach, A., Henke, A., Erberhardt, W., Reichelt, W. and Dettmer, D. (1992) K-Ionregulation in Retina. Canadian Journal of Physiology and Pharmacology, 70, S239-S247. http://dx.doi.org/10.1139/y92-267

[59] Therien, A.G. and Blostein, R. (2000) Mechanisms of Sodium Pump Regulation. American Journal of Physiology-Cell Physiology, 279, C541-C566.

[60] Tasaki, I. (2008) On the Reversible Abrupt Structural Changes in Nerve Fibers Underlying Their Excitation and Conduction Processes. In: Pollack, G.-H. and Chin, W.-C., Eds., Phase Transitions in Cell Biology, Springer, Dordrecht, 121. http://dx.doi.org/10.1007/978-1-4020-8651-9_1 
Scientific Research Publishing (SCIRP) is one of the largest Open Access journal publishers. It is currently publishing more than 200 open access, online, peer-reviewed journals covering a wide range of academic disciplines. SCIRP serves the worldwide academic communities and contributes to the progress and application of science with its publication.

Other selected journals from SCIRP are listed as below. Submit your manuscript to us via either submit@scirp.org or Online Submission Portal.
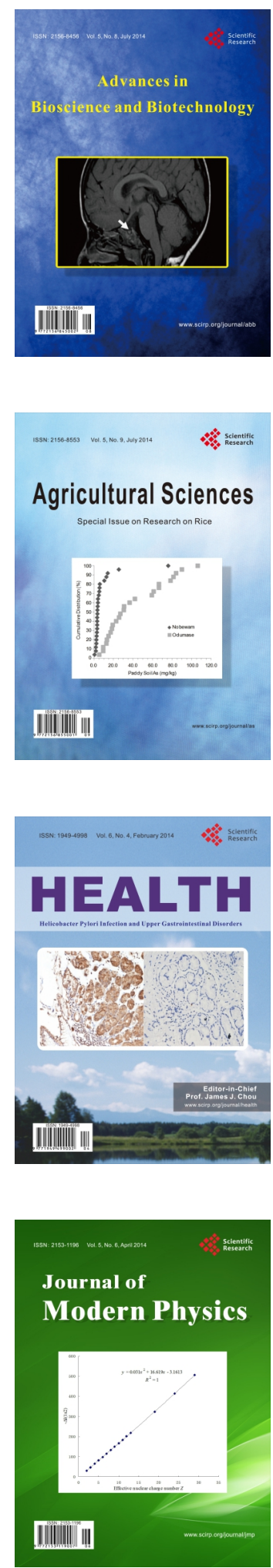
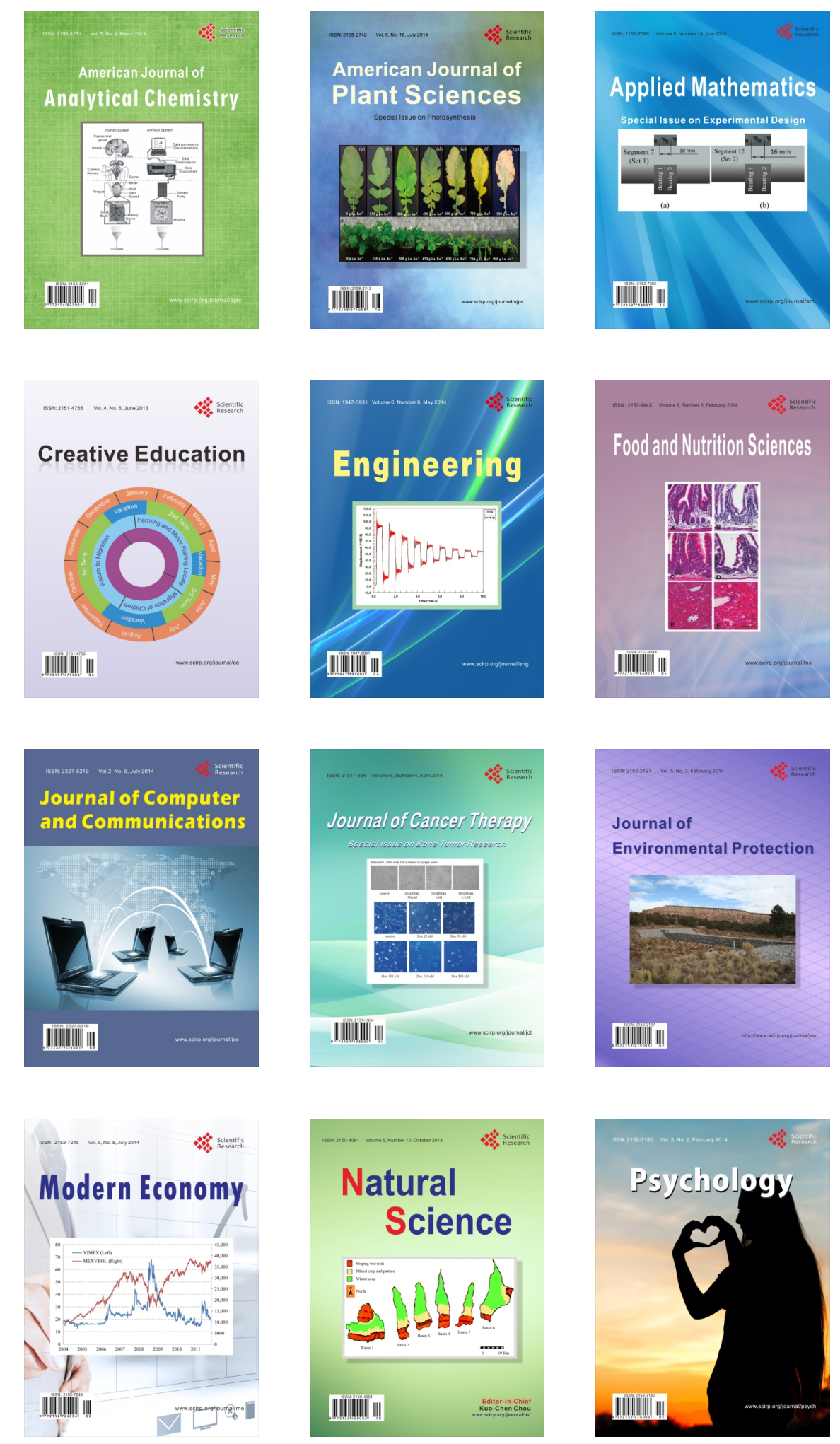